Supporting Information

\title{
Strategies for the Synthesis of 2-Substituted Indoles and Indolines Starting from Acyclic $\alpha$-Phosphonoxy Enecarbamates
}

\author{
Haruhiko Fuwa* and Makoto Sasaki*
}

Laboratory of Biostructural Chemistry, Graduate School of Life Sciences, Tohoku University, 1-1 Tsutsumidori-amamiya, Aoba-ku, Sendai 981-8555, Japan

Email: hfuwa@bios.tohoku.ac.jp

\section{General remarks}

All reactions were performed using oven-dried glasswares under an atmosphere of argon. Anhydrous THF was purchased from Kanto Chemicals Co. Inc. and distilled from $\mathrm{Na}$ /benzophenone immediately prior to use. Anhydrous DMF was purchased from Kanto Chemicals, Co. Inc. and used as received. $\mathrm{CH}_{3} \mathrm{CN}$, methanol, and toluene were distilled from $\mathrm{CaH}_{2}$ under an atmosphere of argon. HMPA was distilled from $\mathrm{CaH}_{2}$ under reduced pressure. Tetrakis(triphenylphosphine)palladium(0) was prepared according to the literature procedure (D. R. Coulson, Inorg. Synth., 1972, 13, 121) and stored under argon at $-20{ }^{\circ} \mathrm{C}$. All other chemicals were purchased at the highest commercial grade and used as received. Analytical thin-layer chromatography (TLC) was performed using E. Merck silica gel $60 \mathrm{~F}_{254}$ pre-coated glass plates $(0.25$ mm-thickness). Flash column chromatography was carried out using Fuji Silysia silica gel BW-300 (200-400 mesh). ${ }^{1} \mathrm{H}$ and ${ }^{13} \mathrm{C}$ NMR spectra were recorded on a Varian Unity INOVA-500 or INOVA-600 spectrometer. Chemical shift values are reported in $\delta$ (ppm) downfield from tetramethylsilane with reference to internal residual solvent $\left[{ }^{1} \mathrm{H}\right.$ NMR: $\mathrm{CHCl}_{3}$ (7.24); ${ }^{13} \mathrm{C} \mathrm{NMR:} \mathrm{CDCl}_{3}$ (77.0)]. Coupling constants $(J)$ are reported in hertz $(\mathrm{Hz})$. Multiplicities of signals are designated as follows: $\mathrm{s}=$ singlet; $\mathrm{d}=$ doublet; $\mathrm{t}=$ triplet; $\mathrm{m}=$ multiplet; $\mathrm{br}=$ broad. Mass spectra were measured on a JEOL JMS-700 spectrometer.

Representative experimental procedures for the synthesis of $\mathbf{6 a}$.

To a solution of imide 1 (131.9 mg, $0.4201 \mathrm{mmol})$ in THF (6 mL) were added HMPA $(0.220 \mathrm{~mL}, 1.26 \mathrm{mmol})$ and diphenylphosphoryl chloride $(0.105 \mathrm{~mL}, 0.507 \mathrm{mmol})$. The mixture was cooled to $-78{ }^{\circ} \mathrm{C}$ and treated with potassium bis(trimethylsilyl)amide 
(0.5 M solution in toluene, $1.00 \mathrm{~mL}, 0.500 \mathrm{mmol})$. After being stirred at $-78^{\circ} \mathrm{C}$ for 30 min, the reaction mixture was treated with $3 \% \mathrm{NH}_{4} \mathrm{OH}$ and diluted with diethyl ether. The resultant mixture was allowed to warm to room temperature over $20 \mathrm{~min}$. The aqueous layer was extracted with EtOAc, and the organic layer was washed with brine, dried $\left(\mathrm{Na}_{2} \mathrm{SO}_{4}\right)$, filtered, and concentrated under reduced pressure. The crude $\alpha$-phosphonoxy enecarbamate thus prepared was used immediately without further purification.

To a solution of the above material in DMF $(5 \mathrm{~mL})$ were added cesium carbonate (3 $\mathrm{M}$ aqueous solution, $0.420 \mathrm{~mL}, 1.26 \mathrm{mmol}$ ), phenylboronic acid (56.4 mg, $0.463 \mathrm{mmol}$ ), and $\mathrm{Pd}\left(\mathrm{PPh}_{3}\right)_{4}(48.5 \mathrm{mg}, 0.0420 \mathrm{mmol})$. The resultant mixture was heated at $50{ }^{\circ} \mathrm{C}$ for $7 \mathrm{~h}$. After cooling, the reaction mixture was diluted with EtOAc, washed with $\mathrm{H}_{2} \mathrm{O}$ and brine, dried $\left(\mathrm{Na}_{2} \mathrm{SO}_{4}\right)$, filtered, and concentrated under reduced pressure. Purification of the residue by flash chromatography (silica gel, 10\% diethyl ether/hexane) gave enecarbamate $\mathbf{6 a}(140.9 \mathrm{mg}, 90 \%)$ as a colorless clear oil.

\section{Representative experimental procedures for the synthesis of 7.}

A heavy-wall pyrex bottle was placed with a solution of 6 a $(118.6 \mathrm{mg}, 0.3180 \mathrm{mmol})$ in DMF $(5 \mathrm{~mL})$. To this solution were added $\mathrm{Et}_{3} \mathrm{~N}(0.440 \mathrm{~mL}, 3.16 \mathrm{mmol})$ and $\mathrm{Pd}\left(\mathrm{PPh}_{3}\right)_{4}(36.7 \mathrm{mg}, 0.0318 \mathrm{mmol})$. The pyrex bottle was purged with argon, capped, and put into a pre-heated oil bath $\left(100{ }^{\circ} \mathrm{C}\right)$ for $20 \mathrm{~h}$. After cooling, the reaction mixture was diluted with EtOAc, washed with $\mathrm{H}_{2} \mathrm{O}$ and brine, dried $\left(\mathrm{Na}_{2} \mathrm{SO}_{4}\right)$, filtered, and concentrated under reduced pressure. Purification of the residue by flash chromatography (silica gel, 10\% diethyl ether/hexane) gave indole 7 (87.8 $\mathrm{mg}, 94 \%$ ) as a colorless clear oil.

Representative experimental procedures for the synthesis of $18\left(n-B u_{3} S n H, A I B N\right)$

To a solution of $6(98.6 \mathrm{mg}, 0.263 \mathrm{mmol})$ in toluene $(4 \mathrm{~mL})$ were added $n-\mathrm{Bu}_{3} \mathrm{SnH}$ $(0.085 \mathrm{~mL}, 0.32 \mathrm{mmol})$ and AIBN $(8.7 \mathrm{mg}, 0.053 \mathrm{mmol})$. The reaction mixture was heated at $100{ }^{\circ} \mathrm{C}$ for $3.5 \mathrm{~h}$. After cooling, the reaction mixture was evaporated. Purification of the residue by flash chromatography (silica gel, $5 \%$ diethyl ether/hexane) gave indoline $18(63.7 \mathrm{mg}, 82 \%)$ as a colorless solid.

Representative experimental procedures for the synthesis of $\mathbf{1 8}\left(\mathrm{SmI}_{2}, t-\mathrm{BuOH}, \mathrm{HMPA}\right)$

To a solution of $\mathrm{SmI}_{2}(0.1 \mathrm{M}$ solution in THF, $5.94 \mathrm{~mL}, 0.594 \mathrm{mmol})$ and HMPA $(0.41 \mathrm{~mL})$ at room temperature was added a solution of $6(55.4 \mathrm{mg}, 0.149 \mathrm{mmol})$ and $t$-BuOH $(0.057 \mathrm{~mL}, 0.60 \mathrm{mmol})$ in THF $(2 \mathrm{~mL}+1 \mathrm{~mL}$ rinse $)$. After being stirred at 
room temperature for $5 \mathrm{~h}$, the reaction mixture was treated with saturated aqueous $\mathrm{NH}_{4} \mathrm{Cl}$. The resultant mixture was extracted with EtOAc. The organic layer was washed with $\mathrm{H}_{2} \mathrm{O}$, saturated aqueous $\mathrm{Na}_{2} \mathrm{SO}_{3}$, and brine. The organic layer was dried $\left(\mathrm{Na}_{2} \mathrm{SO}_{4}\right)$, filtered, and concentrated under reduced pressure. Purification of the residue by flash chromatography (silica gel, 5\% diethyl ether/hexane) gave indoline 18 (39.3 $\mathrm{mg}, 90 \%)$ as a colorless solid.

Spectroscopic data for newly synthesized compounds 6a, 8-17, 19-22.

Compound 6a: IR (neat) $v_{\max } / \mathrm{cm}^{-1} 2977,1715,1622,1475,1299,1164,1088,768,696$; ${ }^{1} \mathrm{H}$ NMR (500 MHz, $\left.\mathrm{C}_{6} \mathrm{D}_{6}\right) \delta 7.59(\mathrm{~d}, J=7.5 \mathrm{~Hz}, 2 \mathrm{H}), 7.37(\mathrm{~d}, J=8.5 \mathrm{~Hz}, 1 \mathrm{H}), 7.21(\mathrm{~d}$, $J=8.0 \mathrm{~Hz}, 1 \mathrm{H}), 7.15-7.12(\mathrm{~m}, 2 \mathrm{H}), 7.08(\mathrm{dd}, J=7.5,7.5 \mathrm{~Hz}, 1 \mathrm{H}), 6.82(\mathrm{dd}, J=8.5$, $8.0 \mathrm{~Hz}, 1 \mathrm{H}), 6.58(\mathrm{dd}, J=7.5,7.5 \mathrm{~Hz}, 1 \mathrm{H}), 4.97$ (s, 1H), $4.83(\mathrm{~s}, 1 \mathrm{H}), 1.22(\mathrm{~s}, 9 \mathrm{H}) ;{ }^{13} \mathrm{C}$ NMR $\left(125 \mathrm{MHz}, \mathrm{C}_{6} \mathrm{D}_{6}\right) \delta 152.4,149.3,143.4,133.6,129.8,129.0,128.5,128.4(\times 2)$, $128.3,128.2,126.5(\times 2), 124.2,108.1,80.8,27.8(\times 3)$; HRMS (FAB) calcd for $\mathrm{C}_{19} \mathrm{H}_{21} \mathrm{NO}_{2}{ }^{79} \mathrm{Br}\left[(\mathrm{M}+\mathrm{H})^{+}\right] 374.0756$, found 374.0756 .

Compound 8: IR (neat) $v_{\max } / \mathrm{cm}^{-1} 2977,1713,1609,1511,1473,1304,1165,1086$, 1031, 838, 745; ${ }^{1} \mathrm{H}$ NMR (500 MHz, $\left.\mathrm{C}_{6} \mathrm{D}_{6}\right) \delta 7.55(\mathrm{~d}, J=7.0 \mathrm{~Hz}, 2 \mathrm{H}), 7.39(\mathrm{~d}, J=8.0$ $\mathrm{Hz}, 1 \mathrm{H}), 7.25$ (d, $J=8.0 \mathrm{~Hz}, 1 \mathrm{H}), 6.84(\mathrm{dd}, J=8.0,7.5 \mathrm{~Hz}, 1 \mathrm{H}), 6.78(\mathrm{~d}, J=7.0 \mathrm{~Hz}$, 2H), $6.60(\mathrm{dd}, J=8.0,7.5 \mathrm{~Hz}, 1 \mathrm{H}), 5.01(\mathrm{~s}, 1 \mathrm{H}), 4.88(\mathrm{~s}, 1 \mathrm{H}), 3.28(\mathrm{~s}, 3 \mathrm{H}), 1.28(\mathrm{~s}, 9 \mathrm{H})$; ${ }^{13} \mathrm{C}$ NMR $\left(125 \mathrm{MHz}, \mathrm{C}_{6} \mathrm{D}_{6}\right) \delta 16.2,152.6,148.9,143.4,133.6,129.6,128.5(\times 2), 128.4$, 128.2, 127.8, 124.1, $113.9(\times 2), 107.2,80.7,54.8,27.9(\times 3)$; HRMS (EI) calcd for $\mathrm{C}_{20} \mathrm{H}_{22}{ }^{79} \mathrm{BrNO}_{3}\left[\mathrm{M}^{+}\right]$403.0783, found 403.0786.

Compound 9: IR (neat) $v_{\max } / \mathrm{cm}^{-1} 2977,1716,1622,1475,1305,1163,1087,838,761$; ${ }^{1} \mathrm{H}$ NMR $\left(500 \mathrm{MHz}, \mathrm{C}_{6} \mathrm{D}_{6}\right) \delta 7.36-7.32(\mathrm{~m}, 3 \mathrm{H}), 7.15-7.08(\mathrm{~m}, 3 \mathrm{H}), 6.82(\mathrm{dd}, J=8.0$, $7.5 \mathrm{~Hz}, 1 \mathrm{H}), 6.59$ (dd, $J=8.0,7.5 \mathrm{~Hz}, 1 \mathrm{H}), 4.83$ (s, 1H), $4.76(\mathrm{~s}, 1 \mathrm{H}), 1.20(\mathrm{~s}, 9 \mathrm{H}) ;{ }^{13} \mathrm{C}$ NMR (125 MHz, $\left.\mathrm{C}_{6} \mathrm{D}_{6}\right) \delta 152.2,148.1,143.0,134.0,133.7,129.7,128.6(\times 2), 128.5$, 128.3, $127.8(\times 2), 124.1,108.4,81.1,27.8(\times 3)$; HRMS (EI) calcd for $\mathrm{C}_{19} \mathrm{H}_{19}{ }^{35} \mathrm{Cl}^{79} \mathrm{BrNO}_{2}\left[\mathrm{M}^{+}\right]$407.0288, found 407.0289.

Compound 10: IR (neat) $v_{\max } / \mathrm{cm}^{-1} 2925,1714,1625,1473,1301,1165,859,760 ;{ }^{1} \mathrm{H}$ NMR (500 MHz, $\left.\mathrm{C}_{6} \mathrm{D}_{6}\right) \delta 7.47(\mathrm{~m}, 1 \mathrm{H}), 7.35(\mathrm{~d}, J=8.0 \mathrm{~Hz}, 1 \mathrm{H}), 7.21(\mathrm{~d}, J=8.0 \mathrm{~Hz}$, $1 \mathrm{H}), 7.04-6.99(\mathrm{~m}, 3 \mathrm{H}), 6.82(\mathrm{dd}, J=8.0,7.0 \mathrm{~Hz}, 1 \mathrm{H}), 6.59(\mathrm{dd}, J=8.0,7.5 \mathrm{~Hz}, 1 \mathrm{H})$, $4.90(\mathrm{~s}, 1 \mathrm{H}), 4.65(\mathrm{~s}, 1 \mathrm{H}), 2.45(\mathrm{~s}, 3 \mathrm{H}), 1.21(\mathrm{~s}, 9 \mathrm{H}) ;{ }^{13} \mathrm{C} \mathrm{NMR}\left(125 \mathrm{MHz}, \mathrm{C}_{6} \mathrm{D}_{6}\right) \delta$ $152.3,146.6,142.1,139.7,136.2$, 133.5, 130.6, 130.3, 128.7, 128.5, 128.4, 128.3, 125.6, 
124.9, 108.1, 80.7, 27.8 ( $\times 3), 20.6$; HRMS (EI) calcd for $\mathrm{C}_{20} \mathrm{H}_{22}{ }^{79} \mathrm{BrNO}_{2}\left[\mathrm{M}^{+}\right]$ 387.0834 , found 387.0834 .

Compound 11: IR (neat) $v_{\max } / \mathrm{cm}^{-1} 2925,1716,1617,1474,1336,1163,853,700 ;{ }^{1} \mathrm{H}$ NMR (500 MHz, $\left.\mathrm{C}_{6} \mathrm{D}_{6}\right) \delta 7.36(\mathrm{~d}, J=6.5 \mathrm{~Hz}, 1 \mathrm{H}), 7.28(\mathrm{~d}, J=7.0 \mathrm{~Hz}, 1 \mathrm{H}), 7.00(\mathrm{~s}$, $1 \mathrm{H}), 6.78$ (dd, $J=6.5,6.0 \mathrm{~Hz}, 1 \mathrm{H}), 6.58$ (dd, $J=7.5,6.5 \mathrm{~Hz}, 1 \mathrm{H}), 6.44$ (brs, 1H), 6.06 $(\mathrm{m}, 1 \mathrm{H}), 5.39$ (brs, 1H), 5.09 (brs, 1H), 1.37 (s, 9H); $\left.{ }^{13} \mathrm{C} \mathrm{NMR} \mathrm{(125} \mathrm{MHz,} \mathrm{C}_{6} \mathrm{D}_{6}\right) \delta$ $152.4,142.8,142.2$, 139.6, 133.5, 129.2, 128.5, 128.4, 128.3, 124.1, 111.6, 107.9, 80.8, $28.0(\times 3)$ (one carbon missing due to solvent overlap); MS (EI) $\mathrm{m} / \mathrm{z} 363$.

Compound 12: IR (neat) $v_{\max } / \mathrm{cm}^{-1} 2977,1716,1617,1474,1336,1164,853,701 ;{ }^{1} \mathrm{H}$ NMR (500 MHz, $\left.\mathrm{C}_{6} \mathrm{D}_{6}\right) \delta 7.36(\mathrm{~d}, J=8.5 \mathrm{~Hz}, 1 \mathrm{H}), 7.30(\mathrm{~d}, J=8.0 \mathrm{~Hz}, 1 \mathrm{H}), 7.15(\mathrm{~m}$, $1 \mathrm{H}), 6.79$ (dd, $J=7.5,7.0 \mathrm{~Hz}, 1 \mathrm{H}), 6.76(\mathrm{~d}, J=4.5 \mathrm{~Hz}, 1 \mathrm{H}), 6.67$ (dd, $J=4.5,3.5 \mathrm{~Hz}$, $1 \mathrm{H}), 6.57(\mathrm{dd}, J=8.0,7.5 \mathrm{~Hz}, 1 \mathrm{H}), 5.16(\mathrm{~s}, 1 \mathrm{H}), 4.97(\mathrm{~s}, 1 \mathrm{H}), 1.37(\mathrm{~s}, 9 \mathrm{H}) ;{ }^{13} \mathrm{C} \mathrm{NMR}$ $\left(125 \mathrm{MHz}, \mathrm{C}_{6} \mathrm{D}_{6}\right) \delta 152.4,143.1,143.0,133.6,129.4,128.5,128.41,128.36,127.3$, 125.2, 125.0, 124.0, 109.0, 81.0, 27.9 (× 3); HRMS (EI) calcd for $\mathrm{C}_{17} \mathrm{H}_{18}{ }^{79} \mathrm{BrNO}_{2} \mathrm{~S}\left[\mathrm{M}^{+}\right]$ 379.0242 , found 379.0245 .

Compound 13: IR (neat) $v_{\max } / \mathrm{cm}^{-1} 2978,1730,1504,1453,1328,1247,1161,1038$, 811, 747; ${ }^{1} \mathrm{H}$ NMR (500 MHz, $\left.\mathrm{CDCl}_{3}\right) \delta 8.20(\mathrm{~d}, J=8.0 \mathrm{~Hz}, 1 \mathrm{H}), 7.54$ (d, $J=7.5 \mathrm{~Hz}$, $1 \mathrm{H}), 7.35(\mathrm{~d}, J=9.0 \mathrm{~Hz}, 2 \mathrm{H}), 7.31(\mathrm{dd}, J=8.0,7.5 \mathrm{~Hz}, 1 \mathrm{H}), 7.24(\mathrm{~m}, 1 \mathrm{H}), 6.95(\mathrm{~d}, J=$ $9.0 \mathrm{~Hz}, 2 \mathrm{H}), 6.51(\mathrm{~s}, 1 \mathrm{H}), 3.85(\mathrm{~s}, 3 \mathrm{H}), 1.36(\mathrm{~s}, 9 \mathrm{H}) ;{ }^{13} \mathrm{C} \mathrm{NMR}\left(125 \mathrm{MHz}, \mathrm{CDCl}_{3}\right) \delta$ $159.2,150.3,140.4,137.3,129.9(\times 2), 129.2,127.4,124.0,122.8,120.2,115.2,113.2$ $(\times 2), 109.4,83.3,55.3,27.6(\times 3)$; HRMS $(\mathrm{EI})$ calcd for $\mathrm{C}_{26} \mathrm{H}_{21} \mathrm{NO}_{3}\left[\mathrm{M}^{+}\right]$323.1521, found 323.1529 .

Compound 14: IR (neat) $v_{\max } / \mathrm{cm}^{-1} 2979,1733,1452,1325,1224,1160,1024,813,747$; ${ }^{1} \mathrm{H}$ NMR $\left(500 \mathrm{MHz}, \mathrm{CDCl}_{3}\right) \delta 8.21(\mathrm{~d}, J=9.0 \mathrm{~Hz}, 1 \mathrm{H}), 7.56(\mathrm{~d}, J=7.5 \mathrm{~Hz}, 1 \mathrm{H})$, $7.39-7.33(\mathrm{~m}, 5 \mathrm{H}), 7.26(\mathrm{~m}, 1 \mathrm{H}), 6.55(\mathrm{~s}, 1 \mathrm{H}), 1.36(\mathrm{~s}, 9 \mathrm{H}) ;{ }^{13} \mathrm{C}$ NMR $(125 \mathrm{MHz}$, $\left.\mathrm{CDCl}_{3}\right) \delta 150.0,139.1,137.4,133.5,133.4,130.0(\times 2), 129.0,128.0(\times 2), 124.5$, 123.0, 120.5, 115.3, 110.4, 83.7, 27.6 (× 3); HRMS (EI) calcd for $\mathrm{C}_{19} \mathrm{H}_{18}{ }^{35} \mathrm{ClNO}_{2}\left[\mathrm{M}^{+}\right]$ 327.1026 , found 327.1031 .

Compound 15: IR (neat) $v_{\max } / \mathrm{cm}^{-1} 2979,1730,1453,1333,1224,1162,1020,747 ;{ }^{1} \mathrm{H}$ NMR (500 MHz, $\left.\mathrm{CDCl}_{3}\right) \delta 8.29(\mathrm{~d}, J=8.5 \mathrm{~Hz}, 1 \mathrm{H}), 7.57(\mathrm{~d}, J=7.5 \mathrm{~Hz}, 1 \mathrm{H})$, $7.35-7.20(\mathrm{~m}, 6 \mathrm{H}), 6.45(\mathrm{~s}, 1 \mathrm{H}), 2.19(\mathrm{~s}, 3 \mathrm{H}), 1.23(\mathrm{~s}, 9 \mathrm{H}) ;{ }^{13} \mathrm{C}$ NMR $(125 \mathrm{MHz}$, 
$\left.\mathrm{CDCl}_{3}\right) \delta 150.1,139.3,137.2,136.7,135.2,129.7,129.4,129.3,128.0,125.2,124.1$, 122.8, 120.3, 115.5, 109.4, 82.9, 27.4 (× 3), 20.0; HRMS (EI) calcd for $\mathrm{C}_{20} \mathrm{H}_{21} \mathrm{NO}_{2}\left[\mathrm{M}^{+}\right]$ 307.1572 , found 307.1575 .

Compound 16: IR (neat) $v_{\max } / \mathrm{cm}^{-1} 2979,1734,1452,1364,1330,1228,1159,1009$, 810, 743; ${ }^{1} \mathrm{H} \mathrm{NMR}\left(500 \mathrm{MHz}, \mathrm{CDCl}_{3}\right) \delta 8.21(\mathrm{~d}, J=8.5 \mathrm{~Hz}, 1 \mathrm{H}), 7.56(\mathrm{~d}, J=7.5 \mathrm{~Hz}$, $1 \mathrm{H}), 7.52(\mathrm{~s}, 1 \mathrm{H}), 7.34(\mathrm{dd}, J=7.5,6.5 \mathrm{~Hz}, 1 \mathrm{H}), 7.24(\mathrm{dd}, J=8.0,7.5 \mathrm{~Hz}, 1 \mathrm{H}), 6.73(\mathrm{~s}$, $1 \mathrm{H}), 6.55(\mathrm{~d}, J=3.5 \mathrm{~Hz}, 1 \mathrm{H}), 6.47(\mathrm{~m}, 1 \mathrm{H}), 1.45(\mathrm{~s}, 9 \mathrm{H}) ;{ }^{13} \mathrm{C} \mathrm{NMR}\left(125 \mathrm{MHz}, \mathrm{CDCl}_{3}\right)$ $\delta 149.8,147.2,142.3,137.3,129.7,128.6,124.9,122.9,120.8,115.3,111.4,110.9$, $109.4,83.5,27.8$ (× 3); HRMS (EI) calcd for $\mathrm{C}_{17} \mathrm{H}_{17} \mathrm{NO}_{3}\left[\mathrm{M}^{+}\right] 283.1208$, found 283.1210 .

Compound 17: IR (neat) $v_{\max } / \mathrm{cm}^{-1} 2979,1732,1453,1328,1218,1160,746 ;{ }^{1} \mathrm{H}$ NMR $\left(500 \mathrm{MHz}, \mathrm{CDCl}_{3}\right) \delta 8.21(\mathrm{~d}, J=8.5 \mathrm{~Hz}, 1 \mathrm{H}), 7.55(\mathrm{~d}, J=8.0 \mathrm{~Hz}, 1 \mathrm{H}), 7.37(\mathrm{~d}, J=5.5$ $\mathrm{Hz}, 1 \mathrm{H}), 7.34(\mathrm{dd}, J=8.0,8.0 \mathrm{~Hz}, 1 \mathrm{H}), 7.25(\mathrm{dd}, J=7.5,7.0 \mathrm{~Hz}, 1 \mathrm{H}), 7.11$ (d, $J=3.5$ $\mathrm{Hz}, 1 \mathrm{H}), 7.06$ (dd, $J=5.5,3.5 \mathrm{~Hz}, 1 \mathrm{H}), 6.67$ (s, 1H), $1.40(\mathrm{~s}, 9 \mathrm{H}) ;{ }^{13} \mathrm{C} \mathrm{NMR}(125 \mathrm{MHz}$, $\left.\mathrm{CDCl}_{3}\right) \delta 150.0,137.4,135.5,132.6,128.7,127.5,126.5,125.9,124.7,123.0,120.5$, 115.3, 111.9, 83.6, $27.6(\times 3)$; HRMS (EI) calcd for $\mathrm{C}_{17} \mathrm{H}_{17} \mathrm{NO}_{2} \mathrm{~S}\left[\mathrm{M}^{+}\right] 299.0980$, found 299.0981 .

Compound 19: IR (neat) $v_{\max } / \mathrm{cm}^{-1} 2975,1702,1608,1483,1388,1248,1171,1036$, 831, 752; ${ }^{1} \mathrm{H}$ NMR (500 MHz, $\left.\mathrm{CDCl}_{3}\right) \delta 7.89$ (br, $\left.1 \mathrm{H}\right), 7.20(\mathrm{dd}, J=7.5,7.5 \mathrm{~Hz}, 1 \mathrm{H})$, $7.11-7.09(\mathrm{~m}, 3 \mathrm{H}), 6.96(\mathrm{dd}, J=7.5,7.0 \mathrm{~Hz}, 1 \mathrm{H}), 6.80-6.77(\mathrm{~m}, 2 \mathrm{H}), 5.30(\mathrm{br}, 1 \mathrm{H})$, $3.76(\mathrm{~s}, 3 \mathrm{H}), 3.63(\mathrm{dd}, J=16.0,11.0 \mathrm{~Hz}, 1 \mathrm{H}), 2.93$ (dd, $J=16.0,3.0 \mathrm{~Hz}, 1 \mathrm{H}), 1.34$ (br, $9 \mathrm{H}) ;{ }^{13} \mathrm{C}$ NMR $\left(125 \mathrm{MHz}, \mathrm{CDCl}_{3}\right) \delta 158.6,152.4,143.0,137.0,129.4,127.5,126.5(\times$ 2), 124.8, $122.5(\times 2), 114.7,113.7,80.6,62.0,55.2,37.8,28.2(\times 3)$; HRMS (EI) calcd for $\mathrm{C}_{20} \mathrm{H}_{23} \mathrm{NO}_{3}\left[\mathrm{M}^{+}\right]$325.1675, found 325.1680.

Compound 20: IR (neat) $v_{\max } / \mathrm{cm}^{-1} 2976,1703,1484,1387,1278,1167,1015,827,751$; ${ }^{1} \mathrm{H}$ NMR $\left(500 \mathrm{MHz}, \mathrm{CDCl}_{3}\right) \delta 7.91(\mathrm{br}, 1 \mathrm{H}), 7.24-7.20(\mathrm{~m}, 3 \mathrm{H}), 7.12-7.10(\mathrm{~m}, 3 \mathrm{H})$, 6.97 (dd, $J=7.5,7.0 \mathrm{~Hz}, 1 \mathrm{H}), 5.32$ (br, 1H), 3.66 (dd, $J=16.5,10.5 \mathrm{~Hz}, 1 \mathrm{H}), 2.90$ (dd, $J=16.5,2.5 \mathrm{~Hz}, 1 \mathrm{H}), 1.31(\mathrm{br}, 9 \mathrm{H}) ;{ }^{13} \mathrm{C} \mathrm{NMR}\left(125 \mathrm{MHz}, \mathrm{CDCl}_{3}\right) \delta 152.2,143.0,132.7$, $128.6(\times 2), 127.7(\times 2), 126.7(\times 2), 124.8,122.7(\times 2), 114.7,81.1,61.9,37.7,28.1(\times$ 3); HRMS (EI) calcd for $\mathrm{C}_{19} \mathrm{H}_{20}{ }^{35} \mathrm{ClNO}_{2}\left[\mathrm{M}^{+}\right] 329.1179$, found 329.1185 .

Compound 21: IR (neat) $v_{\max } / \mathrm{cm}^{-1} 2974,1703,1484,1389,1168,1016,752 ;{ }^{1} \mathrm{H}$ NMR 
$\left(500 \mathrm{MHz}, \mathrm{CDCl}_{3}\right) \delta 7.98(\mathrm{br}, 1 \mathrm{H}), 7.23(\mathrm{~m}, 1 \mathrm{H}), 7.14-7.01(\mathrm{~m}, 5 \mathrm{H}), 6.96(\mathrm{dd}, J=7.5$, $7.0 \mathrm{~Hz}, 1 \mathrm{H}$ ), 5.57 (br, 1H), 3.68 (dd, $J=16.0,11.0 \mathrm{~Hz}, 1 \mathrm{H}$ ), 2.82 (app. d, $J=16.0 \mathrm{~Hz}$, $1 \mathrm{H}), 2.38(\mathrm{~s}, 3 \mathrm{H}), 1.23(\mathrm{br}, 9 \mathrm{H}) ;{ }^{13} \mathrm{C}$ NMR $\left(125 \mathrm{MHz}, \mathrm{CDCl}_{3}\right) \delta 152.3,143.0(\times 2)$, 133.4, 130.2, 128.9, 127.6, 126.7, 126.3, 124.9, 123.6, 122.5, 114.5, 80.4, 59.2, 36.5, 28.0, $19.3(\times 3)$; HRMS (EI) calcd for $\mathrm{C}_{20} \mathrm{H}_{23} \mathrm{NO}_{2}\left[\mathrm{M}^{+}\right] 309.1725$, found 309.1730 .

Compound 22: IR (neat) $v_{\max } / \mathrm{cm}^{-1} 2976,1703,1483,1387,1320,1278,1167,1014$, 748; ${ }^{1} \mathrm{H}$ NMR $\left(500 \mathrm{MHz}, \mathrm{CDCl}_{3}\right) \delta 7.81(\mathrm{br}, 1 \mathrm{H}), 7.27(\mathrm{~s}, 1 \mathrm{H}), 7.19(\mathrm{dd}, J=6.5,6.5 \mathrm{~Hz}$, $1 \mathrm{H}), 7.15(\mathrm{~d}, J=6.0 \mathrm{~Hz}, 1 \mathrm{H}), 6.96(\mathrm{dd}, J=6.5,6.5 \mathrm{~Hz}, 1 \mathrm{H}), 6.26(\mathrm{~m}, 1 \mathrm{H}), 6.12(\mathrm{~s}, 1 \mathrm{H})$, 5.47 (br, 1H), 3.52 (dd, $J=13.5,9.0 \mathrm{~Hz}, 1 \mathrm{H}), 3.15$ (dd, $J=13.5,2.5 \mathrm{~Hz}, 1 \mathrm{H}), 1.47$ (br, $9 \mathrm{H}) ;{ }^{13} \mathrm{C} \mathrm{NMR}\left(125 \mathrm{MHz}, \mathrm{CDCl}_{3}\right) \delta 155.3,152.1,142.3,141.4,129.4,127.5,124.6$, $122.5,115.0,110.1,105.5,80.9,56.3,34.3,28.2(\times 3)$; HRMS (EI) calcd for $\mathrm{C}_{17} \mathrm{H}_{19} \mathrm{NO}_{3}$ $\left[\mathrm{M}^{+}\right]$285.1354, found 285.1369. 

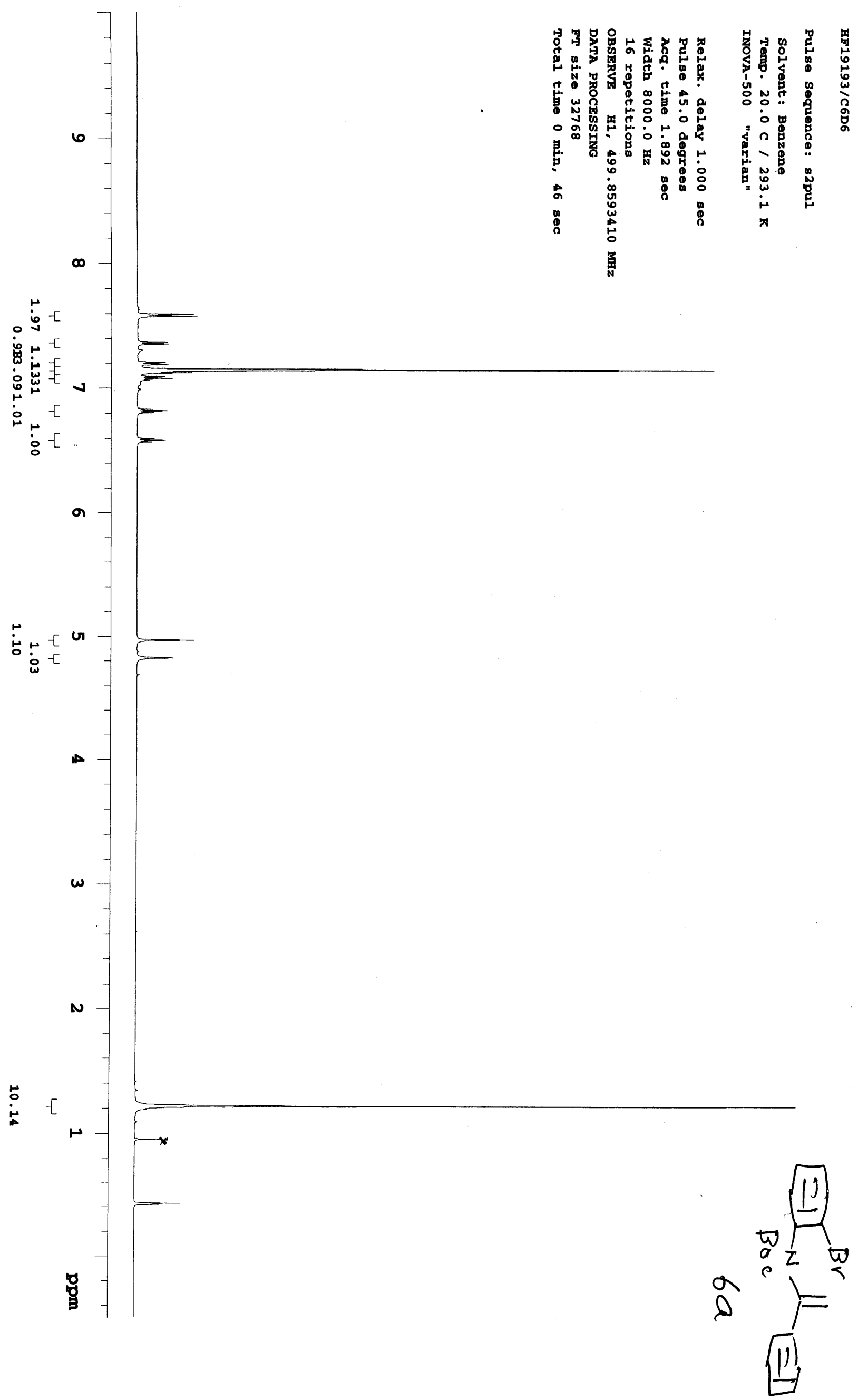


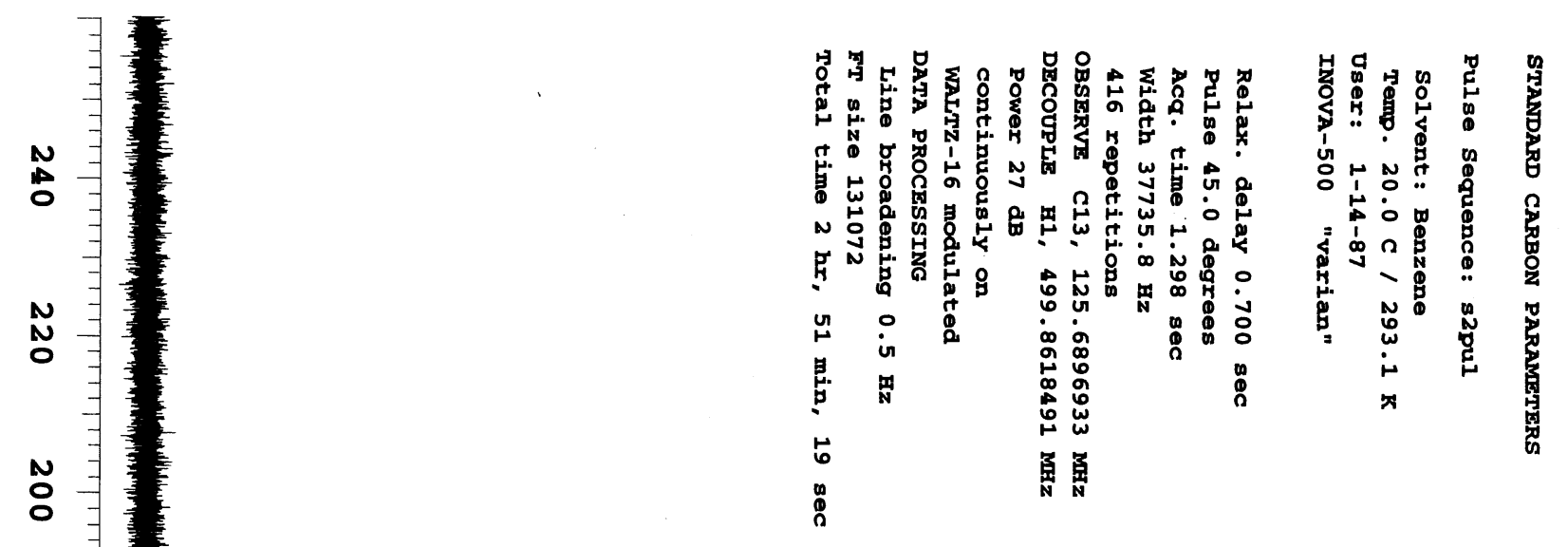

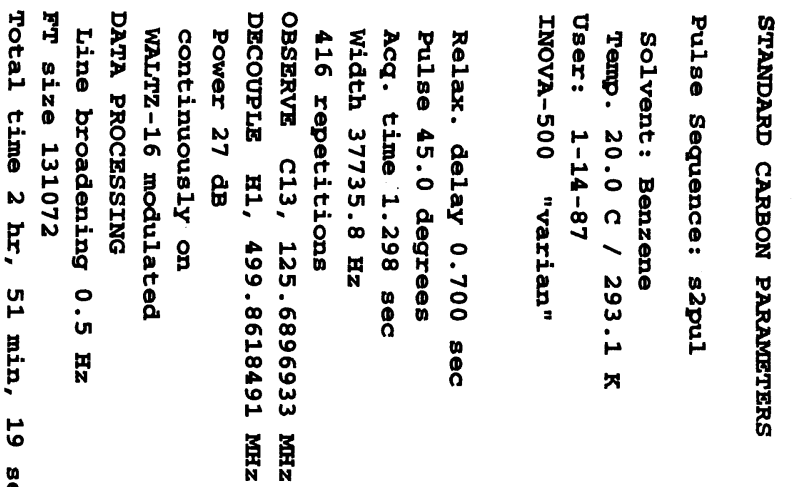

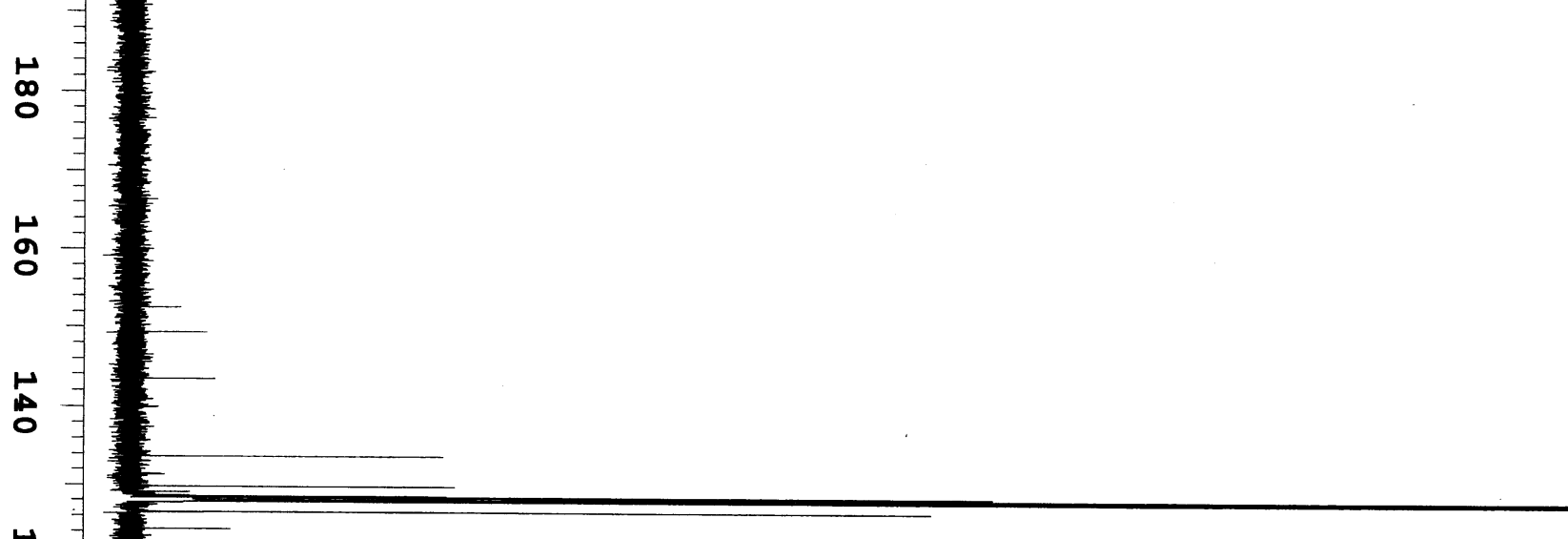

N

怘

$\infty$

。

음

㟔

再

N

N

$\circ$

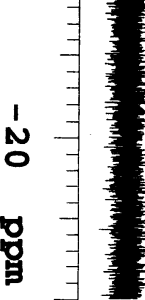

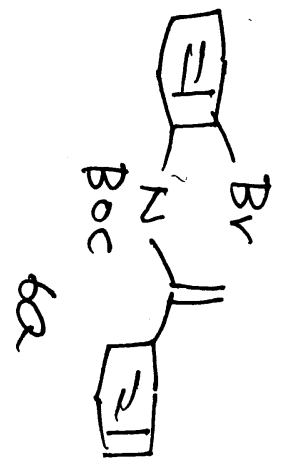



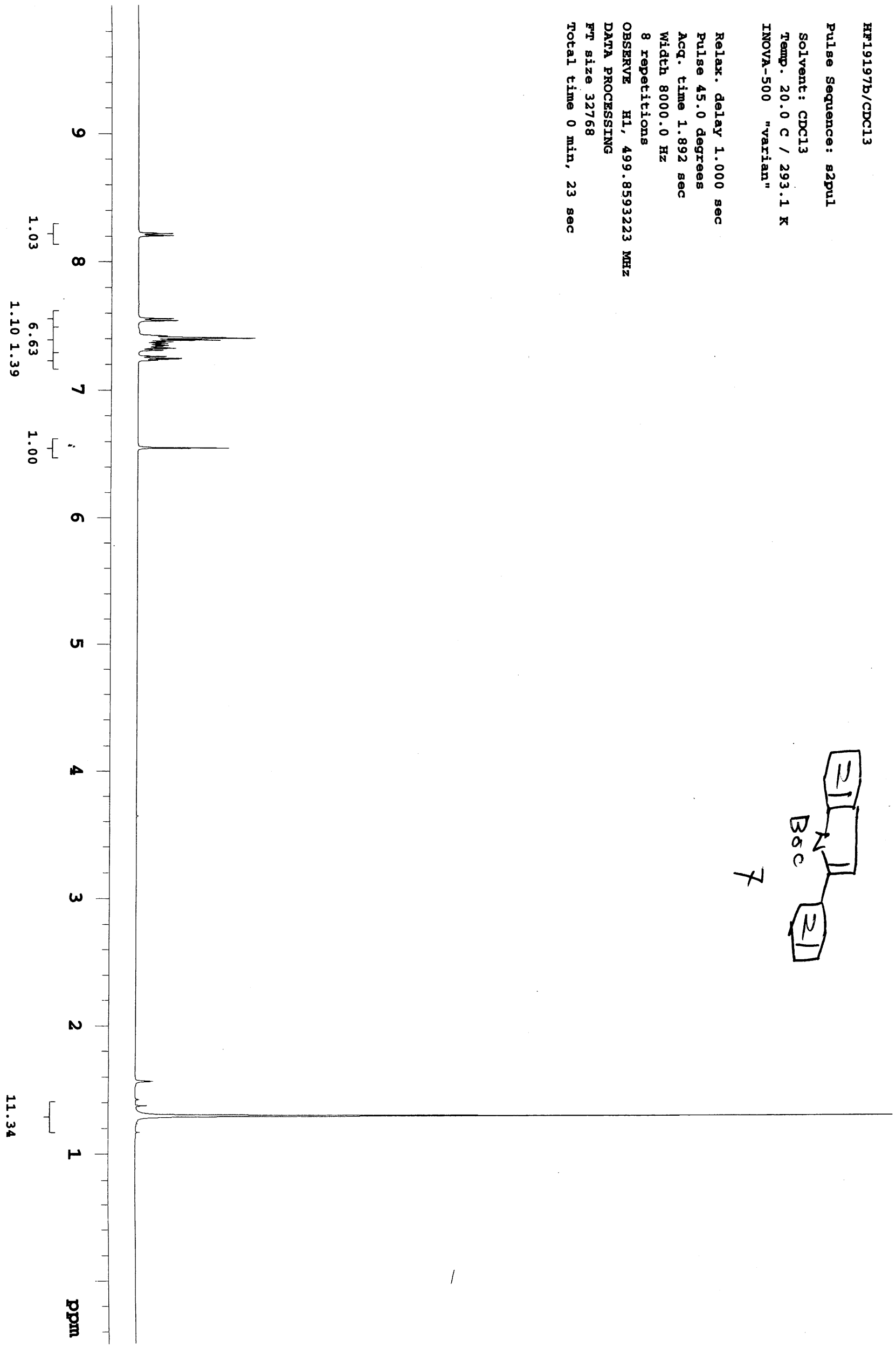


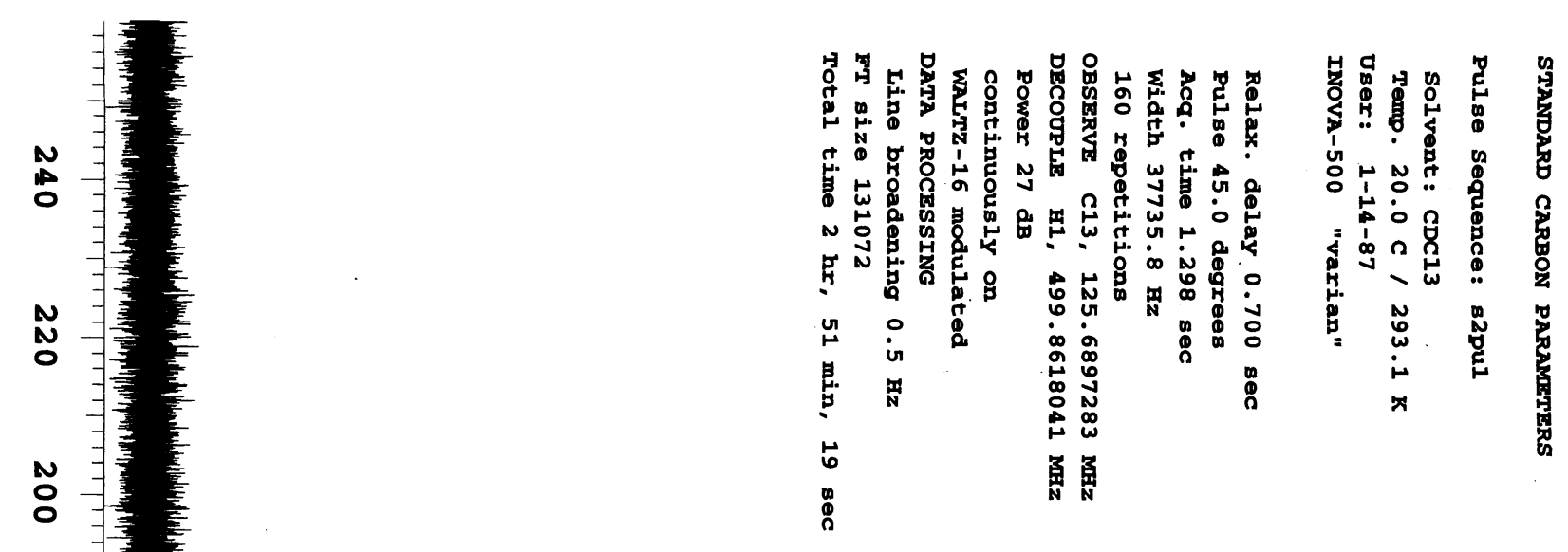

$\stackrel{\infty}{\infty}$

مै

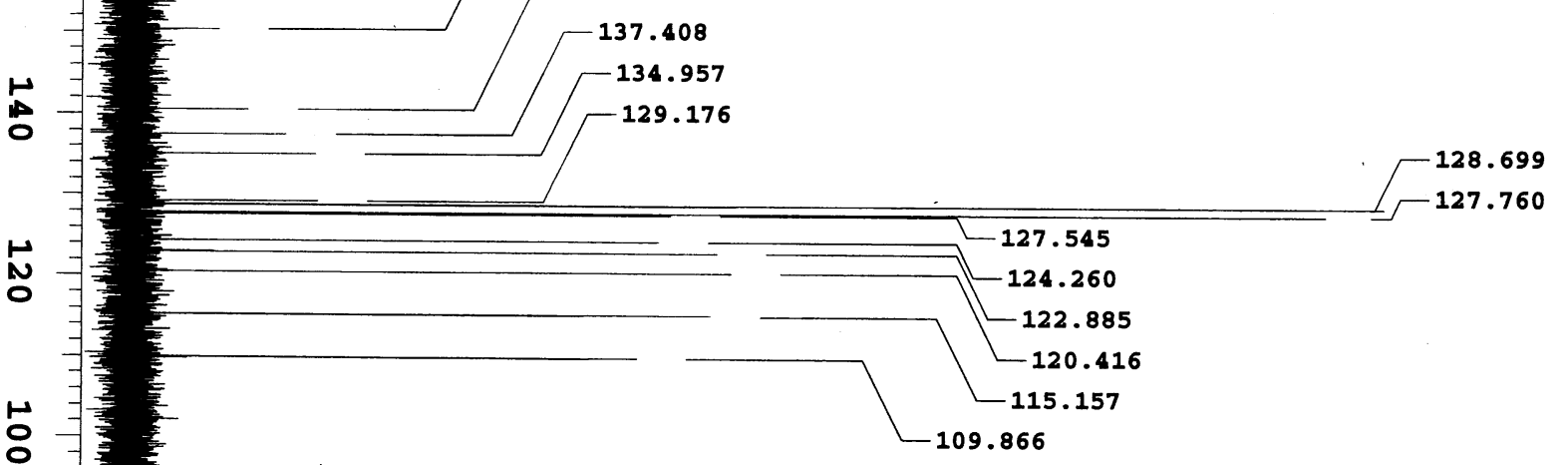

농

$\infty$

$-83.354$

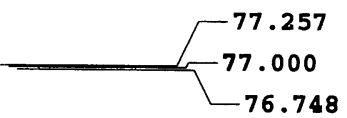

음

○

음

0

,

-

手竞旁

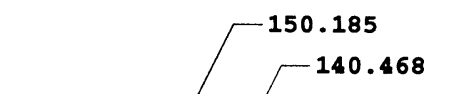

$-137.408$

品

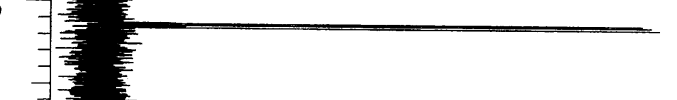

$-76.748$

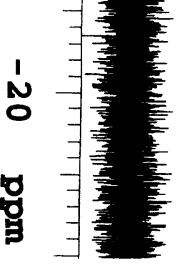

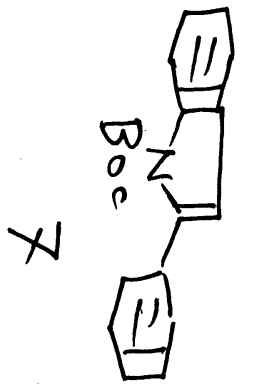




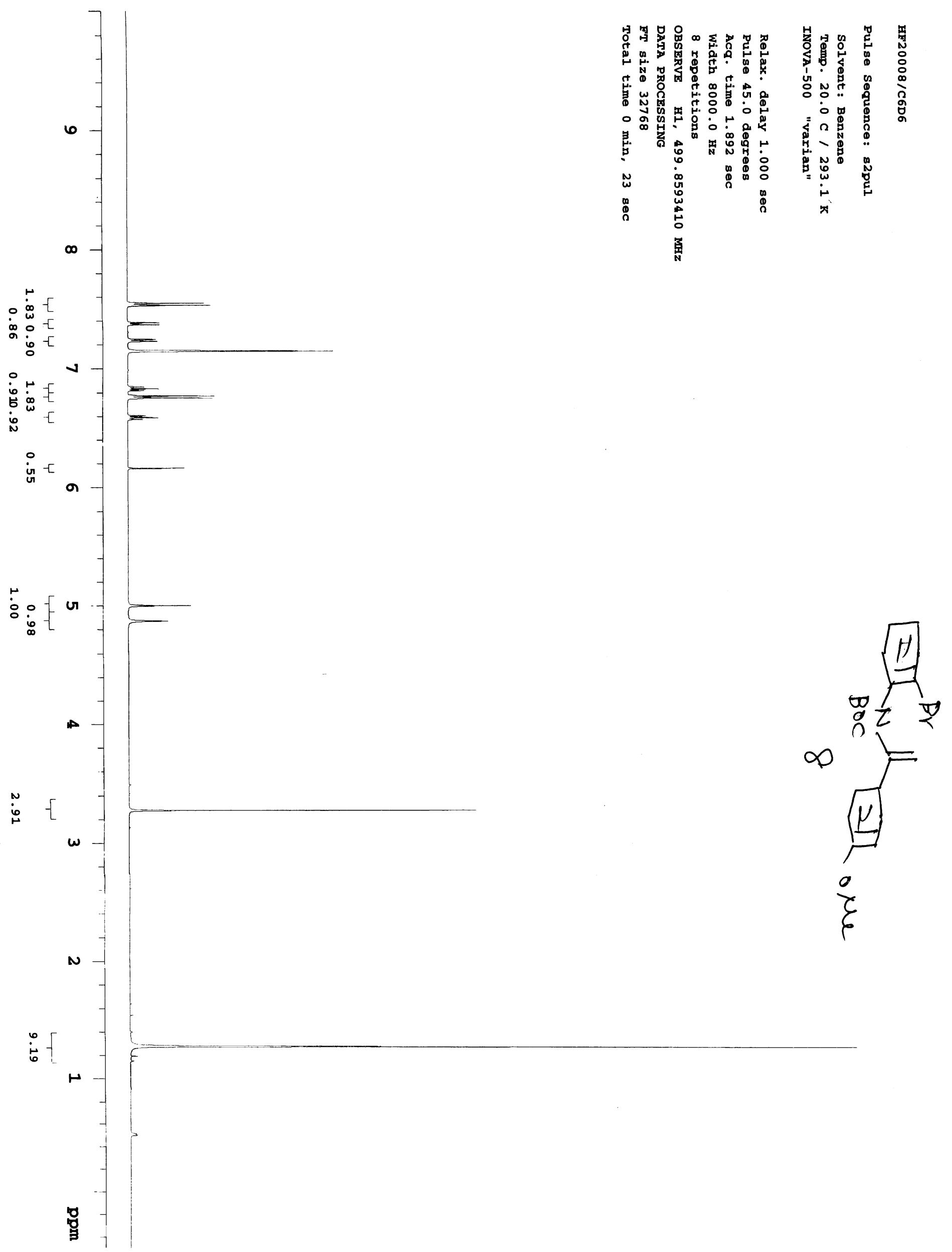



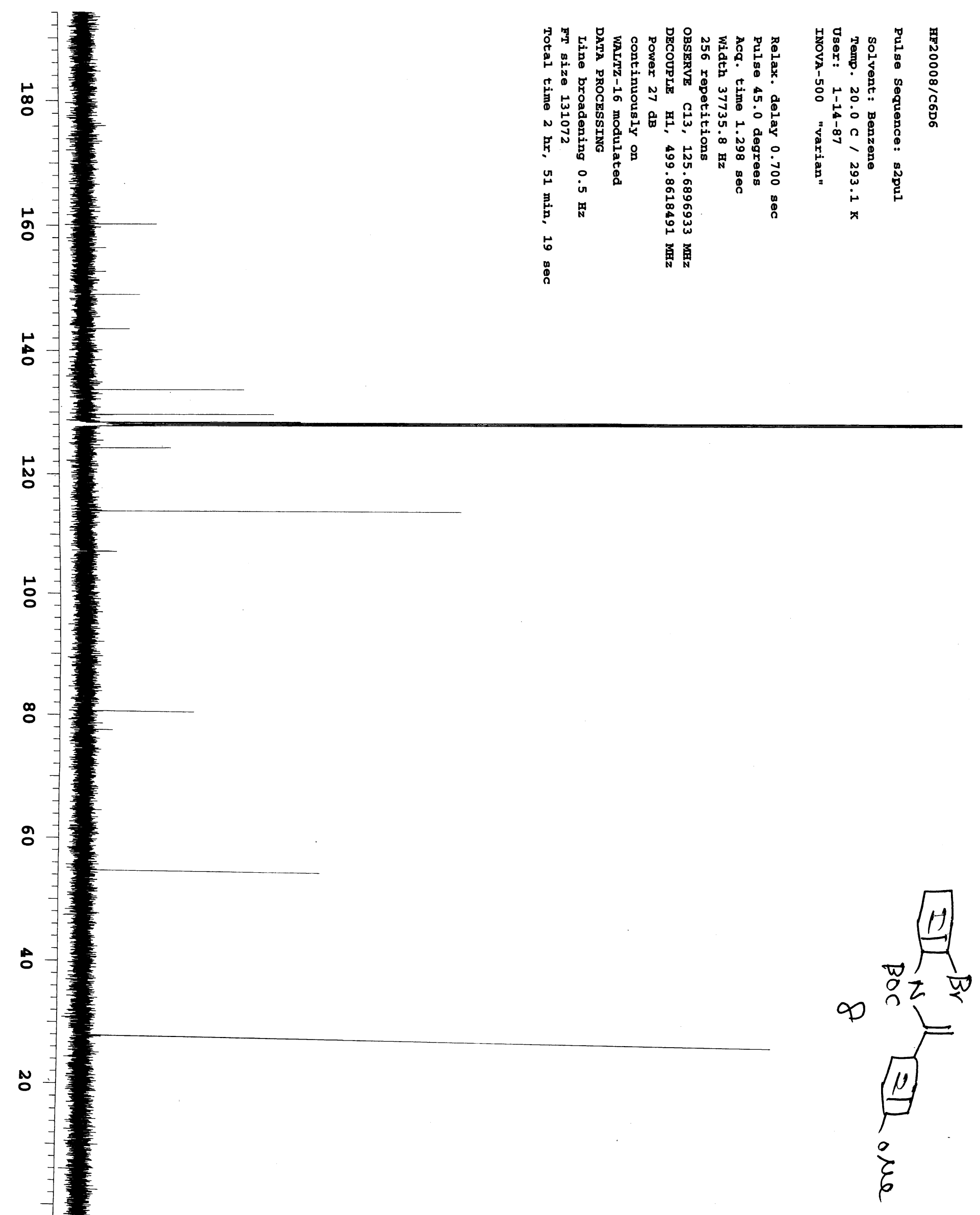


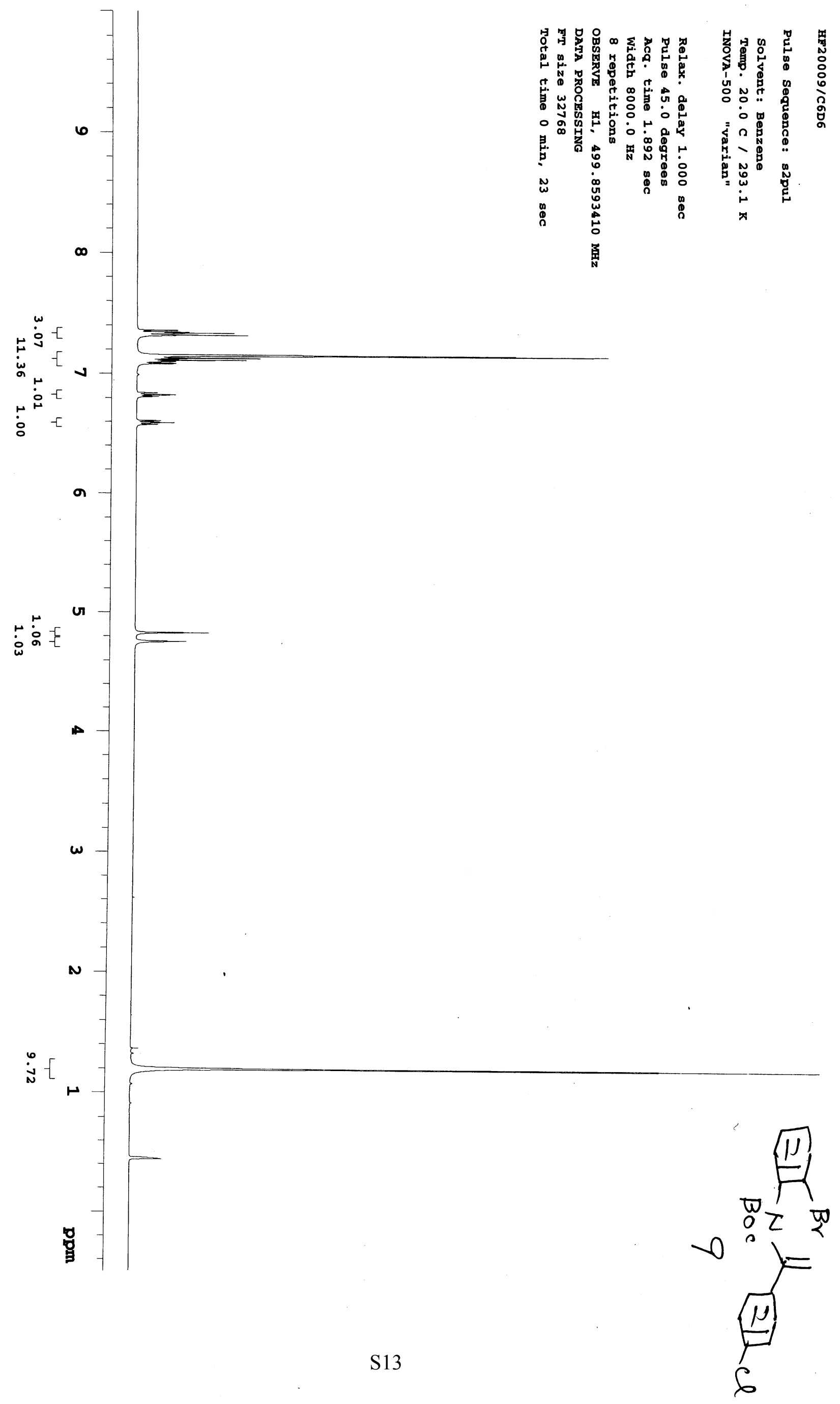



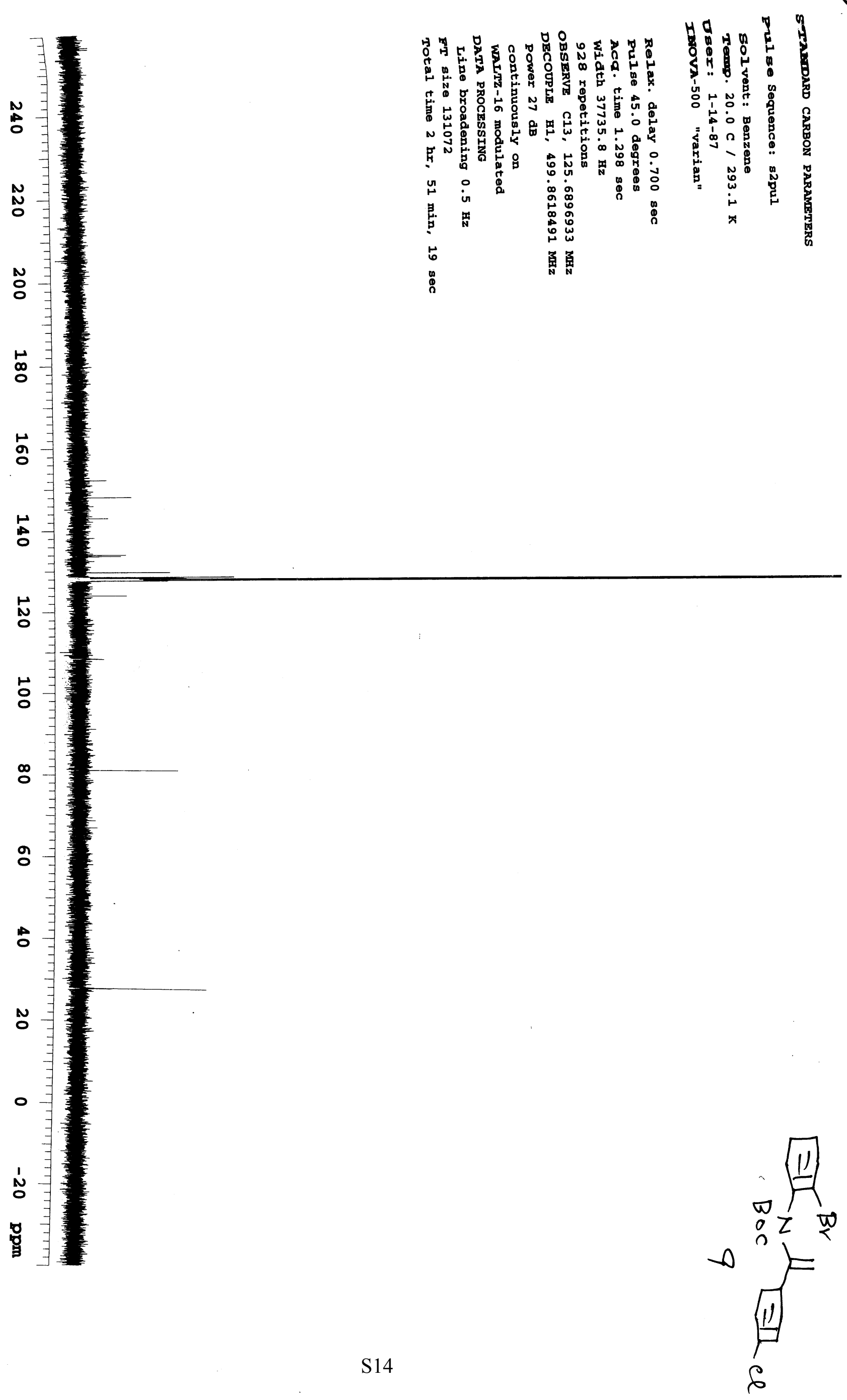


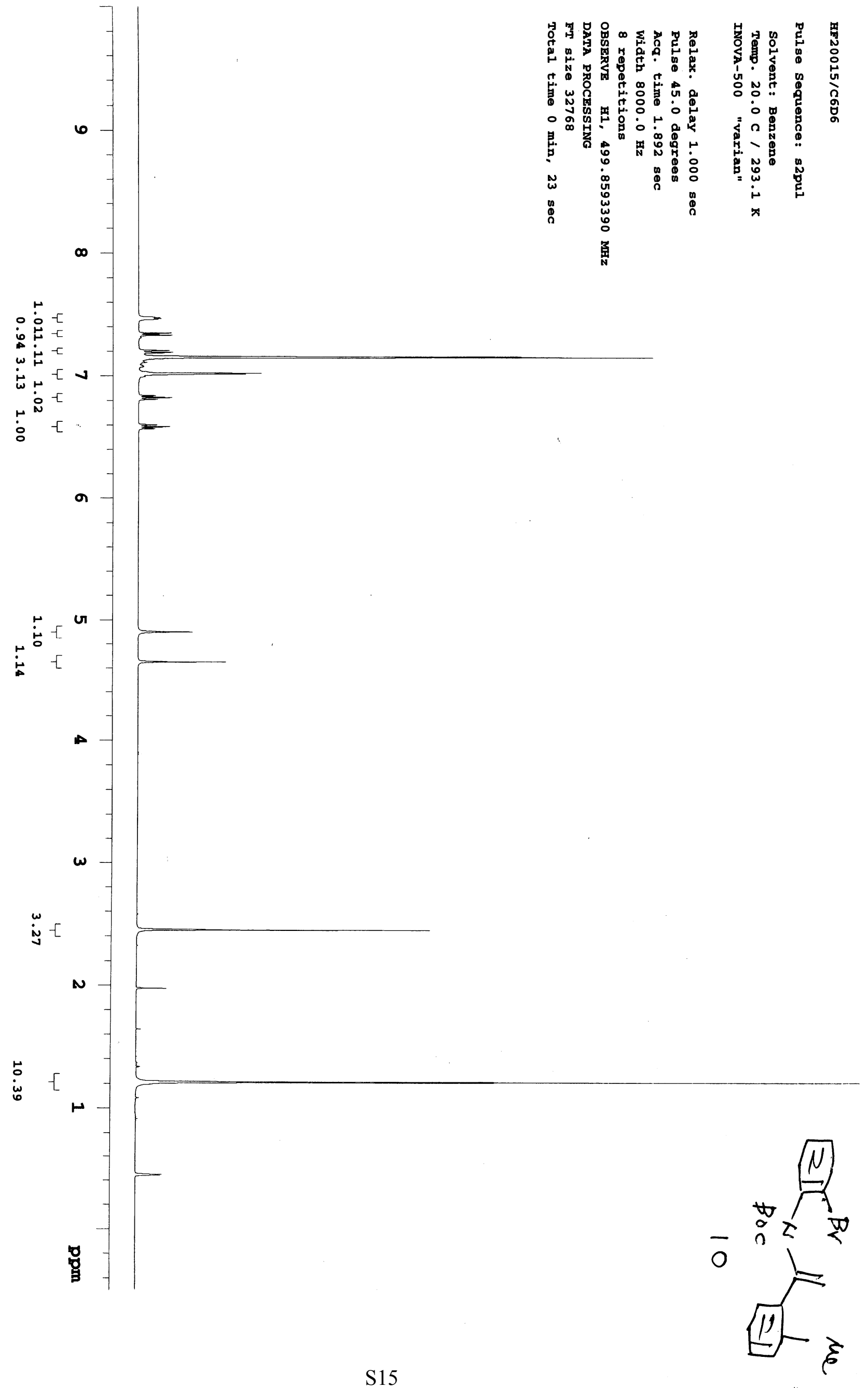




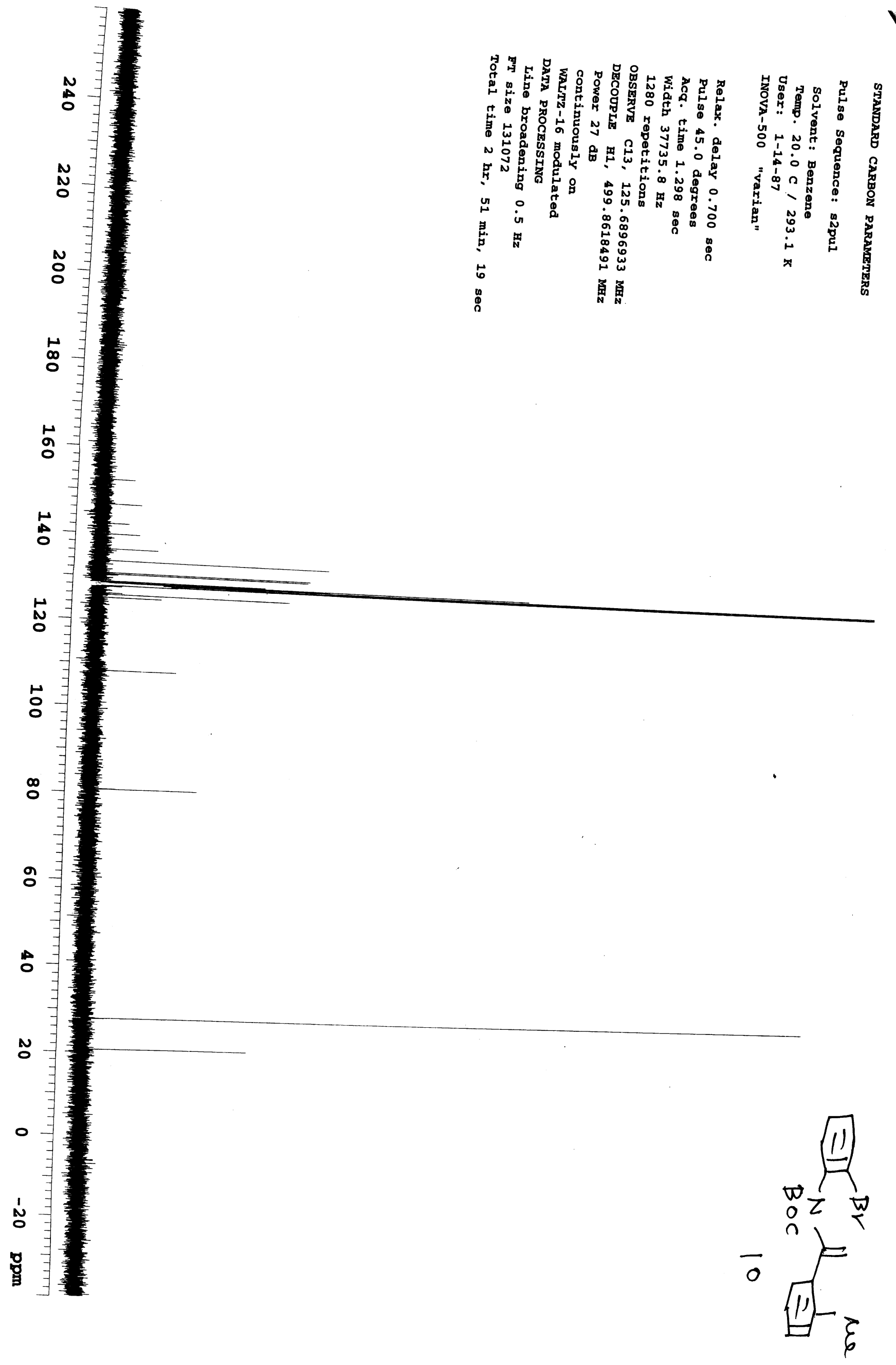




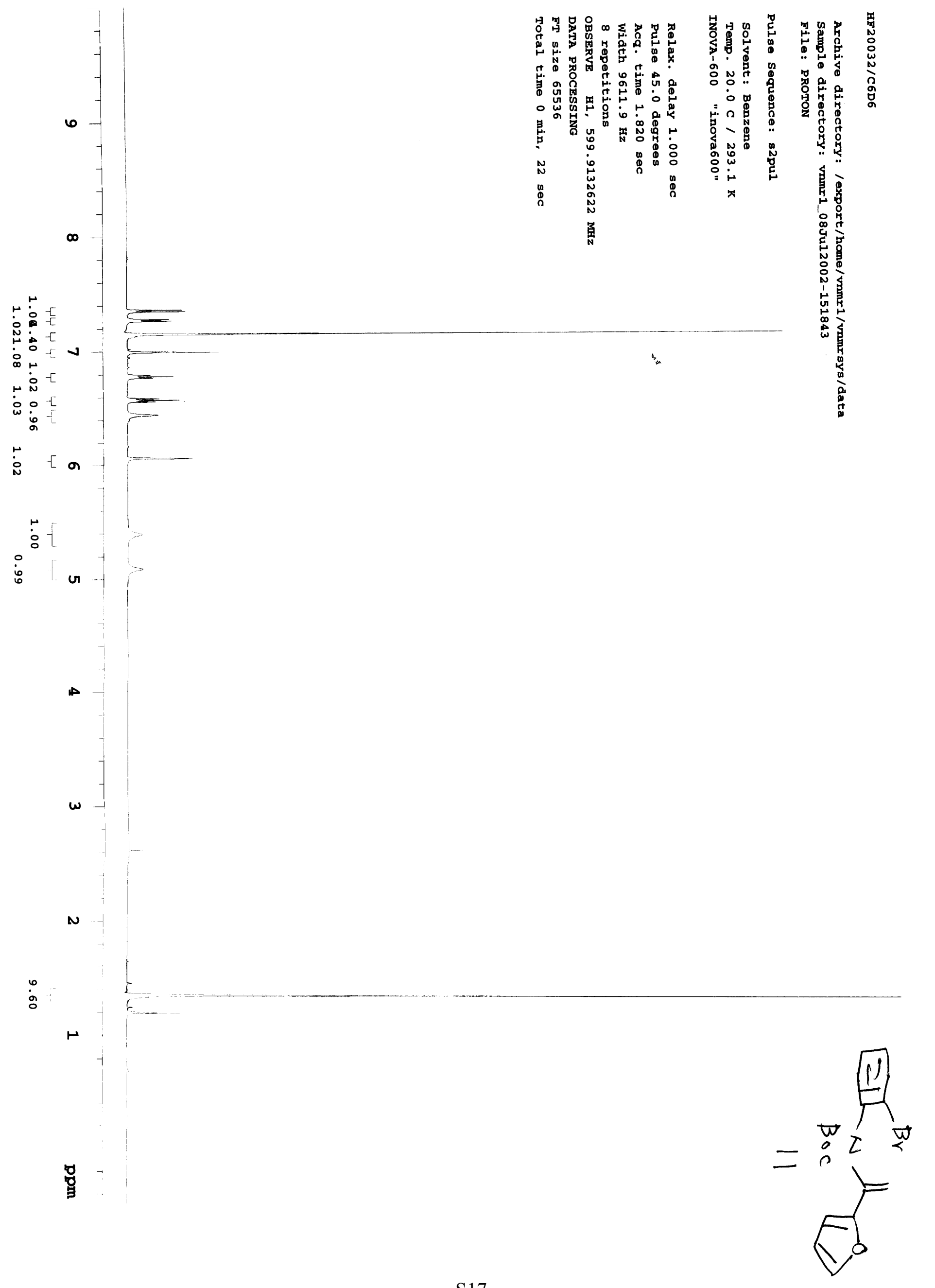




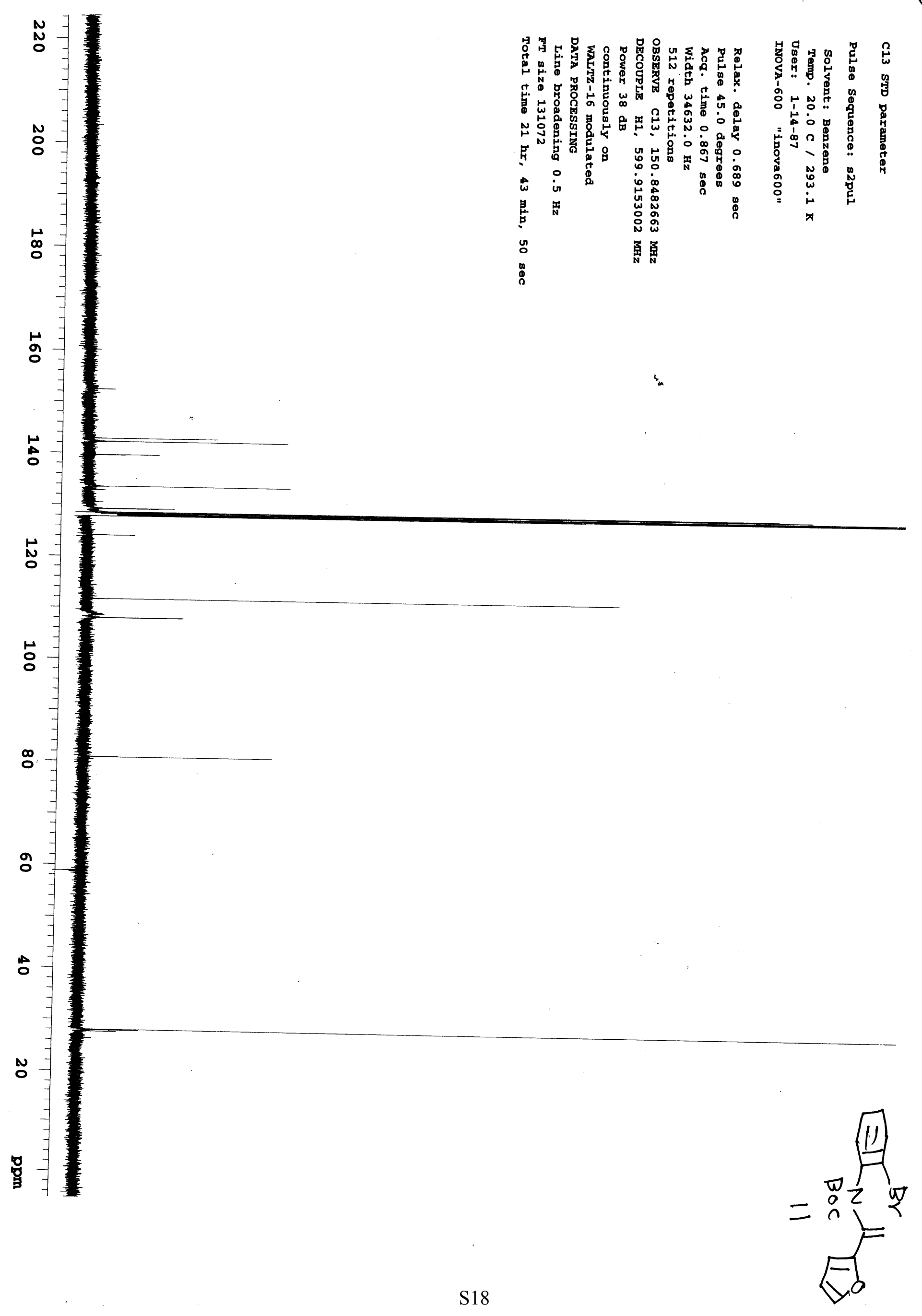




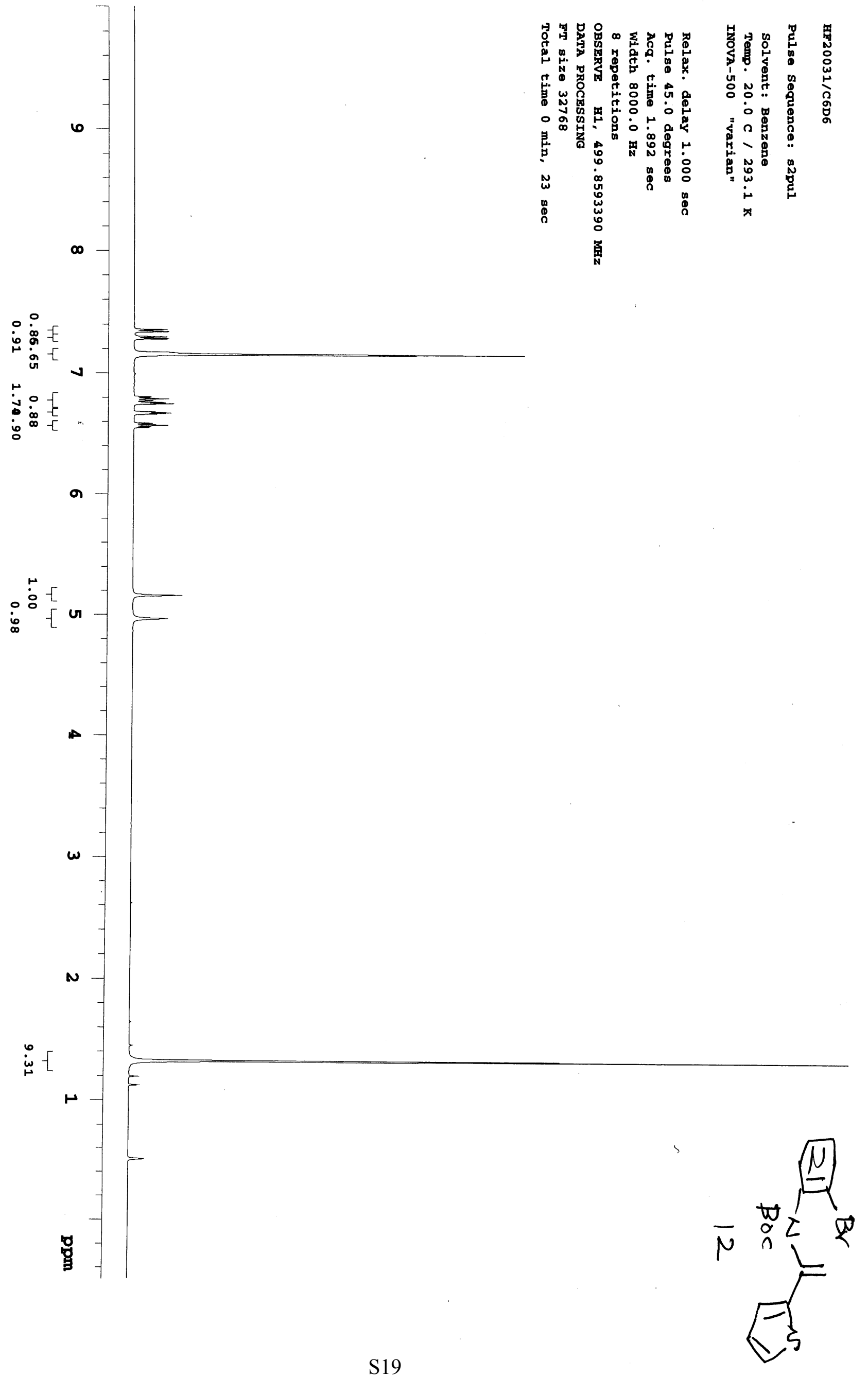




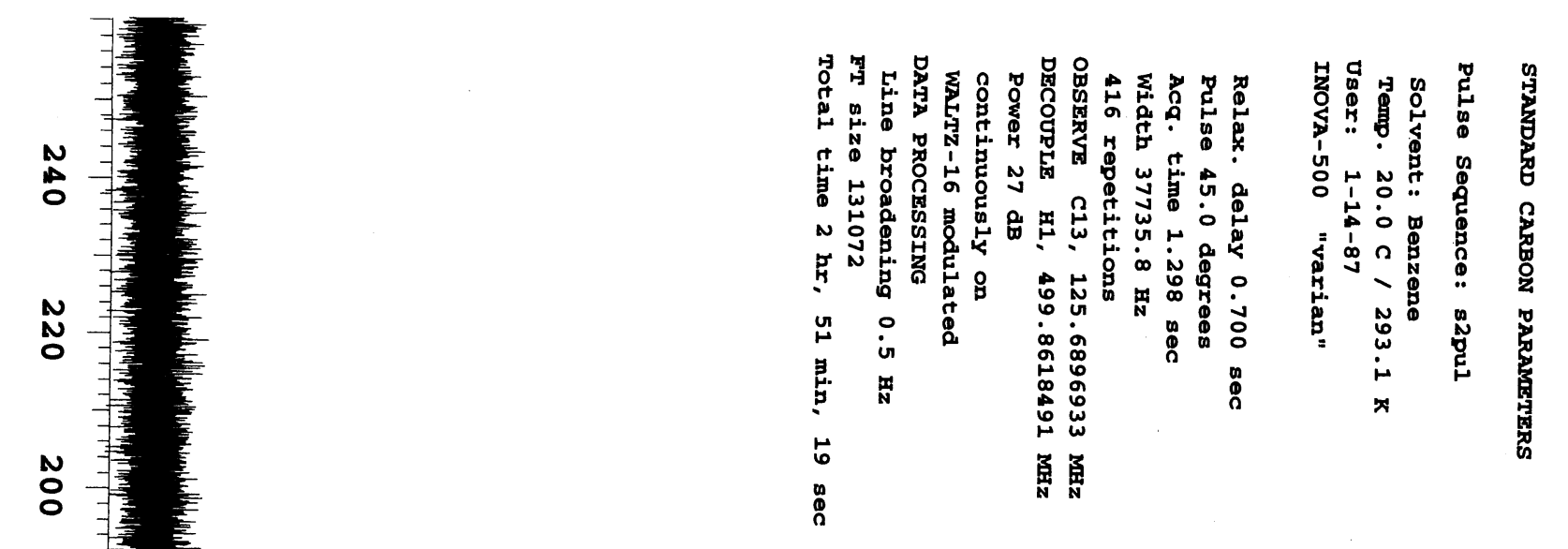

畧

옹

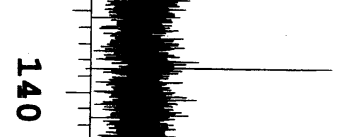

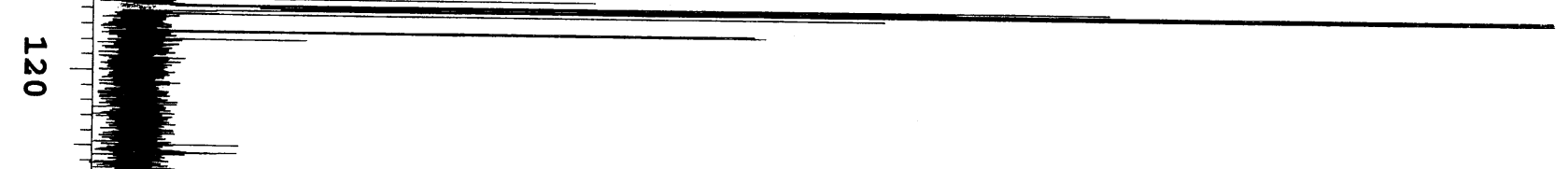

:

$\infty$

g

。氺

을

0

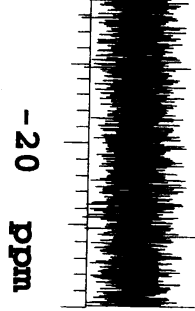

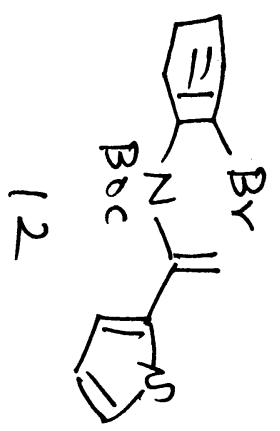




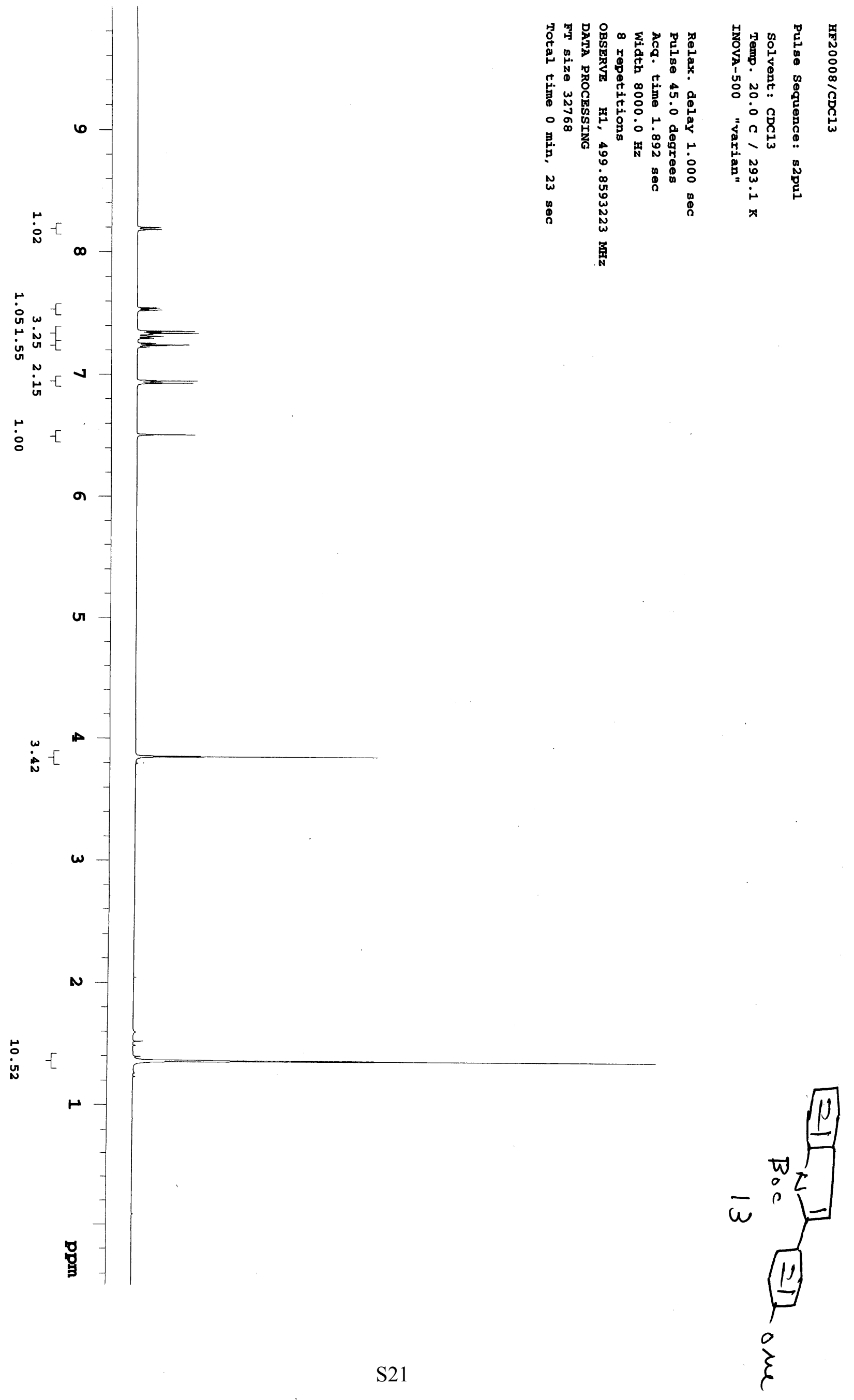




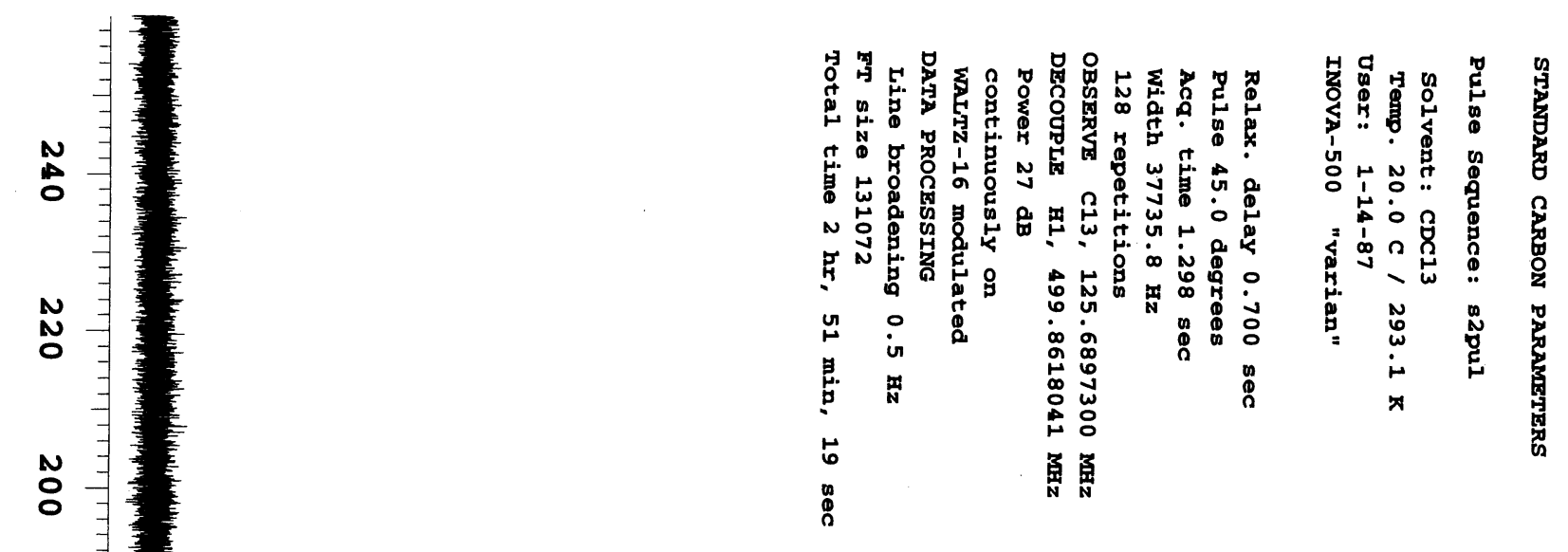

1

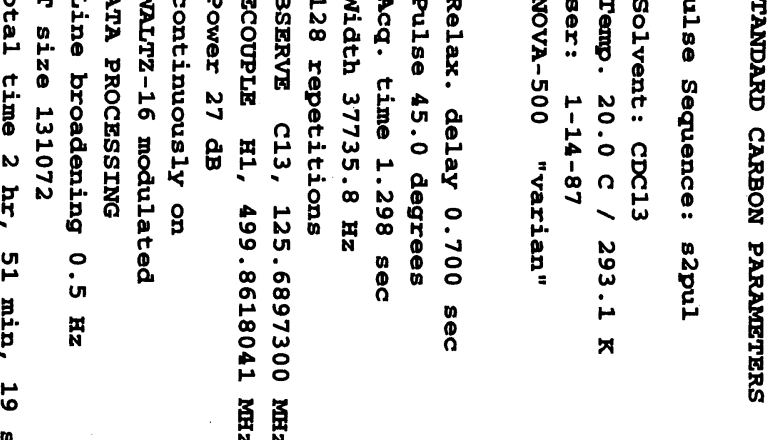

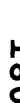

$\stackrel{\infty}{\circ}$

응

占寻售旁旁

N

웅

$\infty$

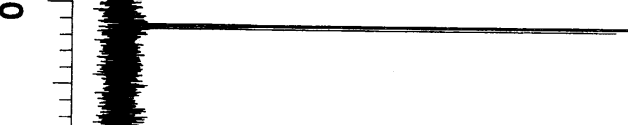

g

\&

o

今

手章

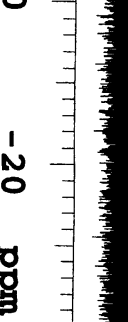

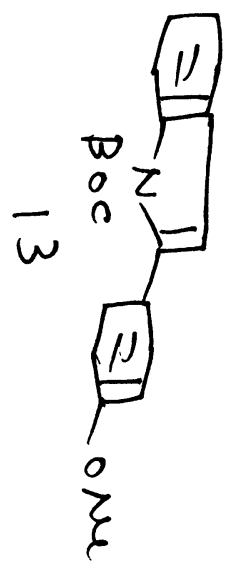




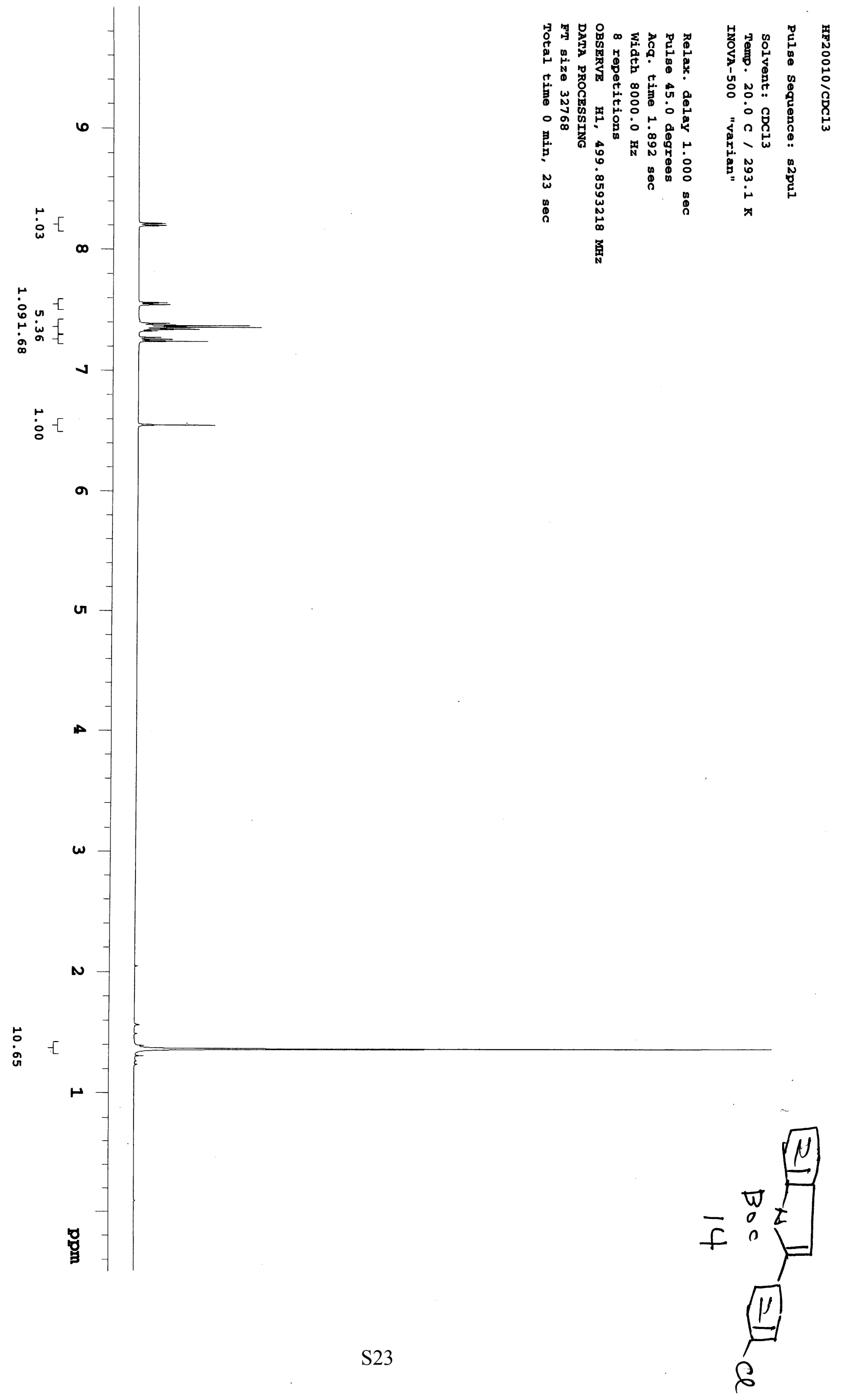



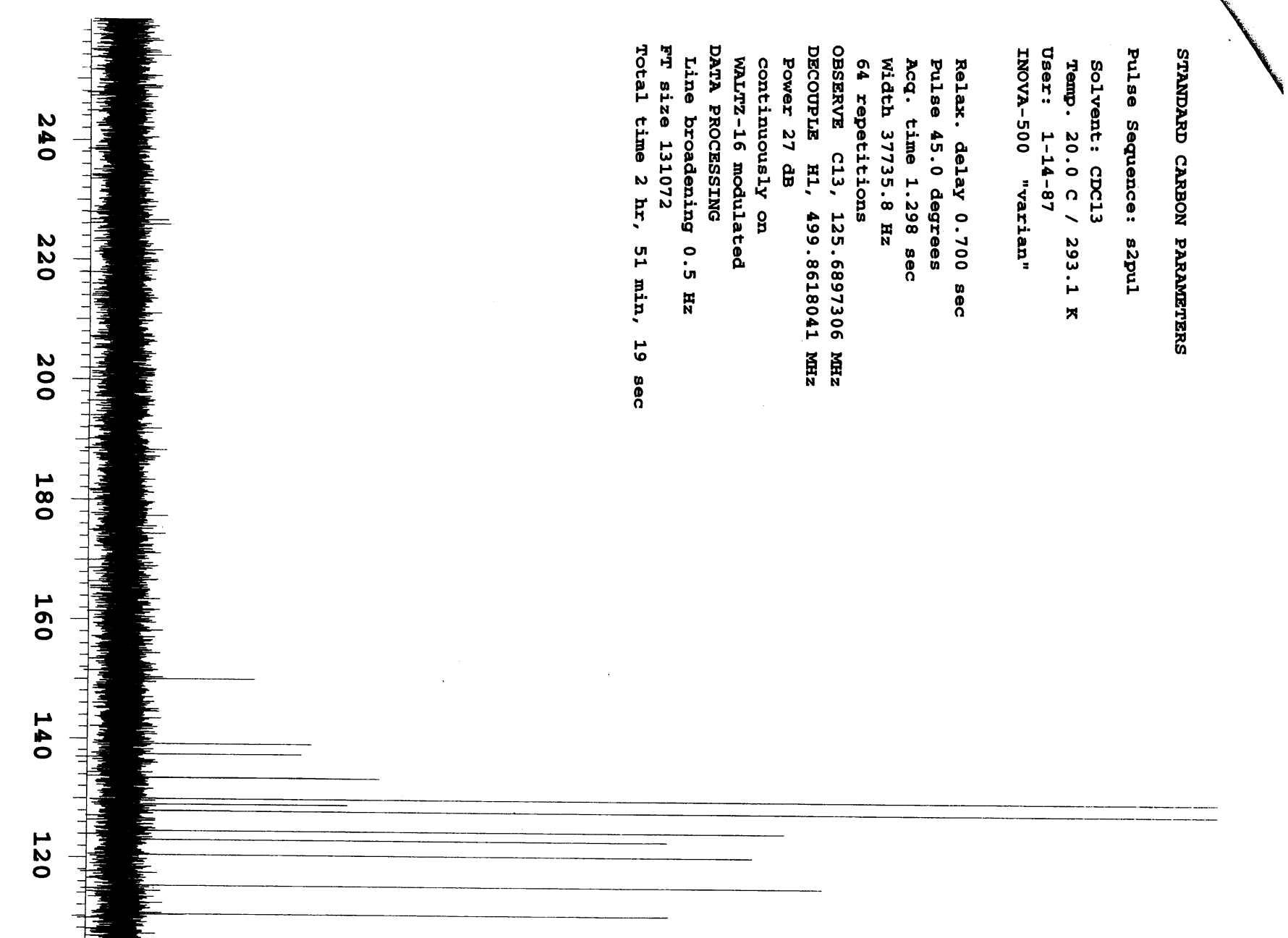

怘

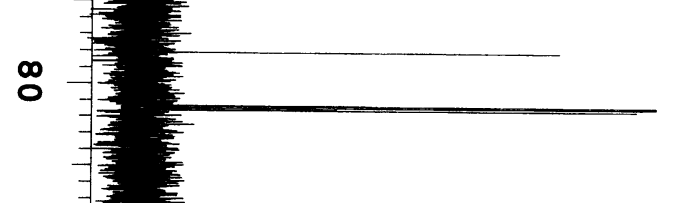

:

吅雍

N

$\circ$

I

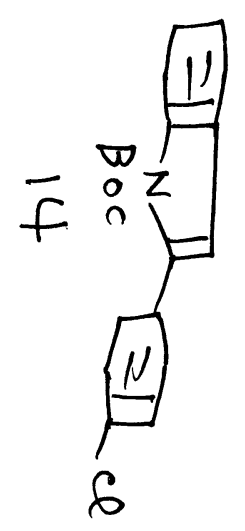




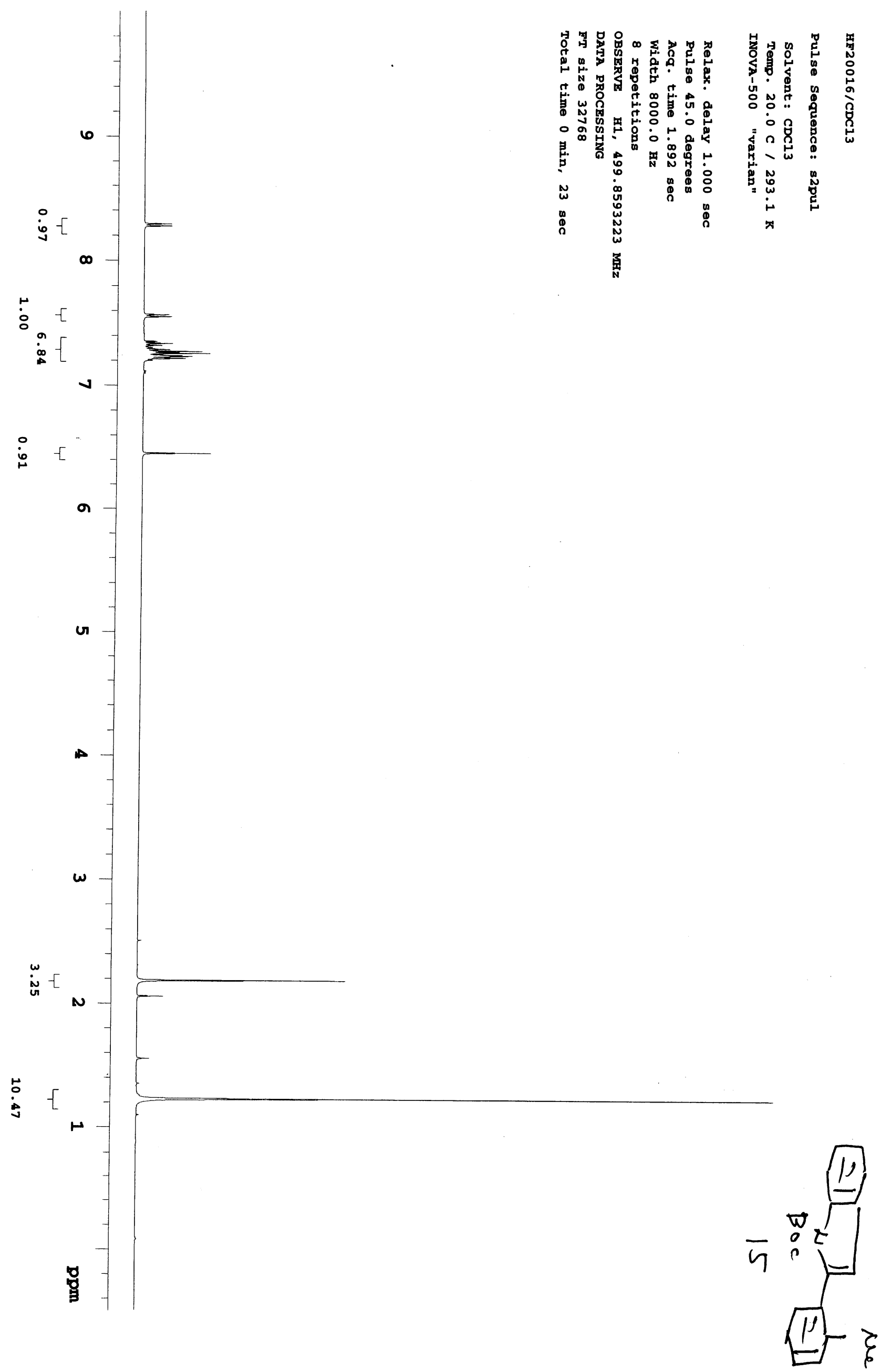




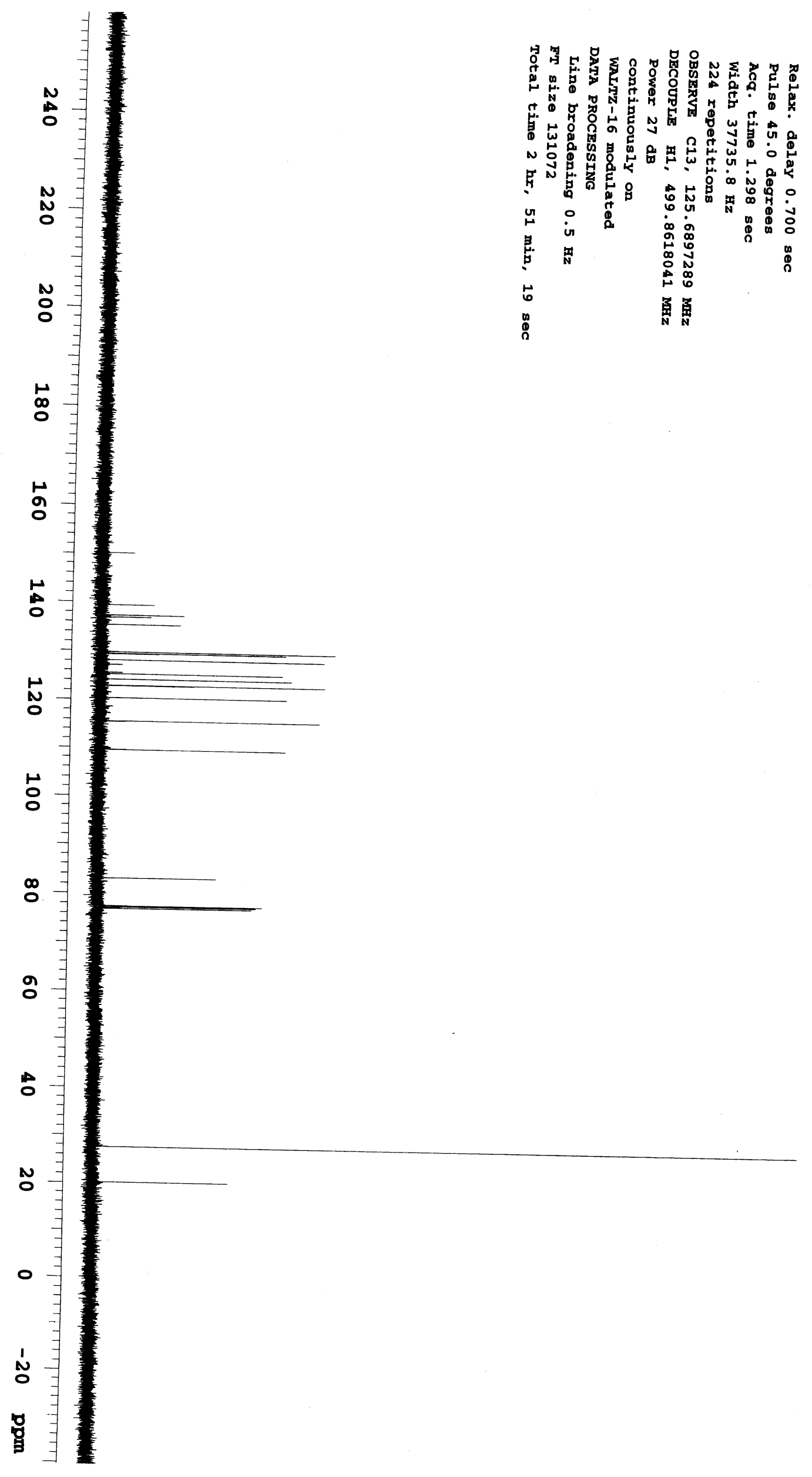

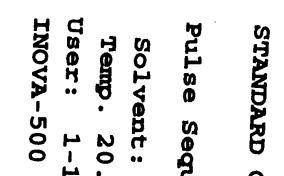

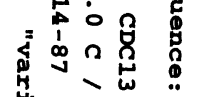

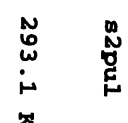

芯壳 


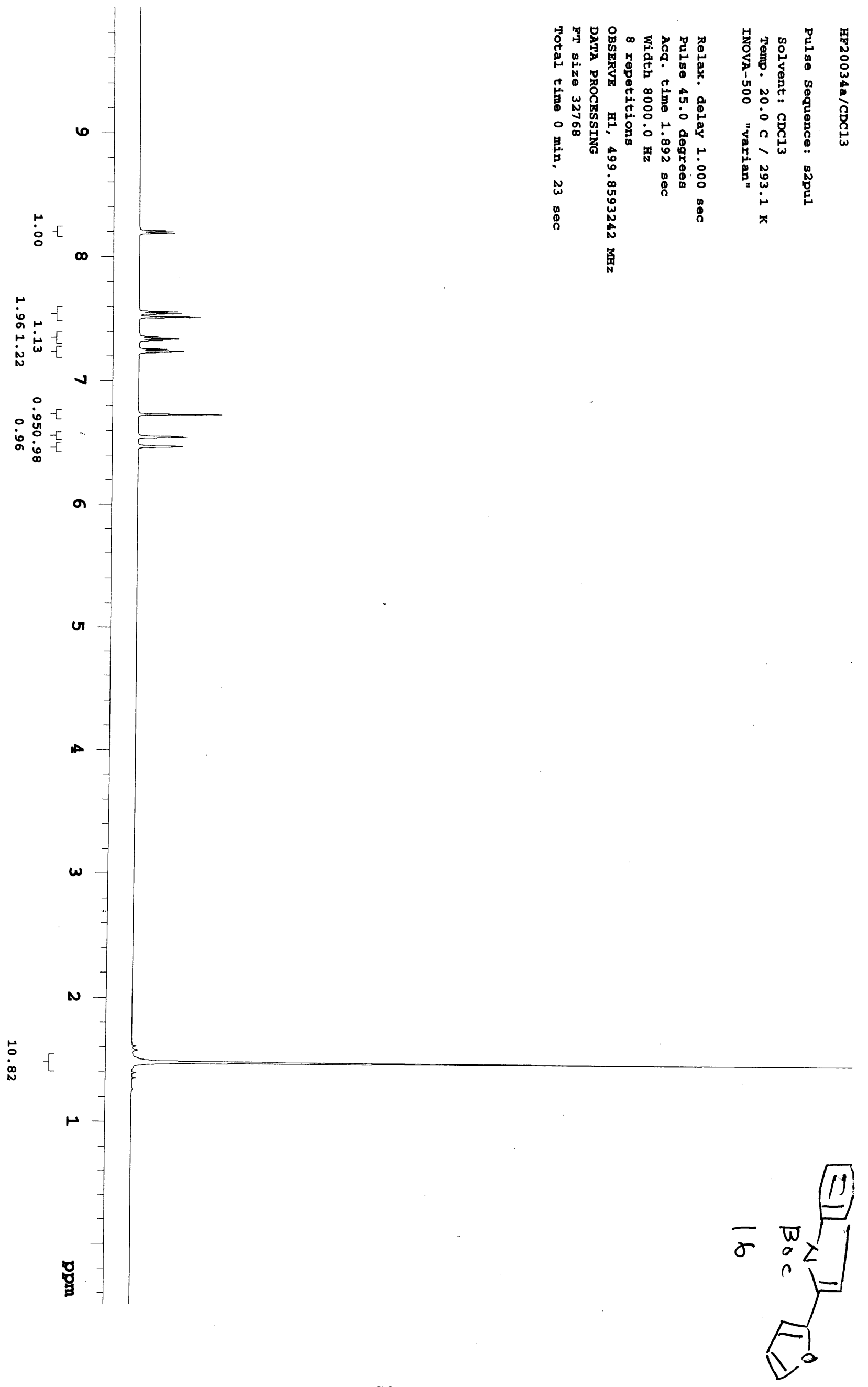




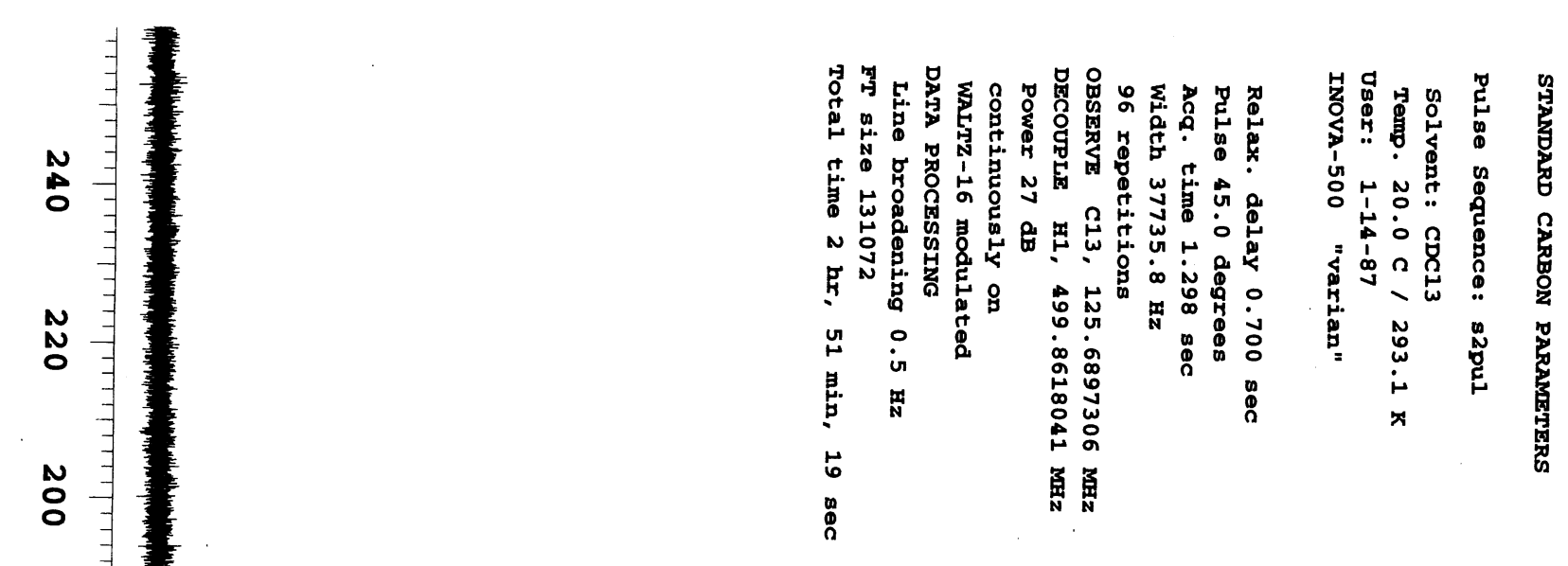

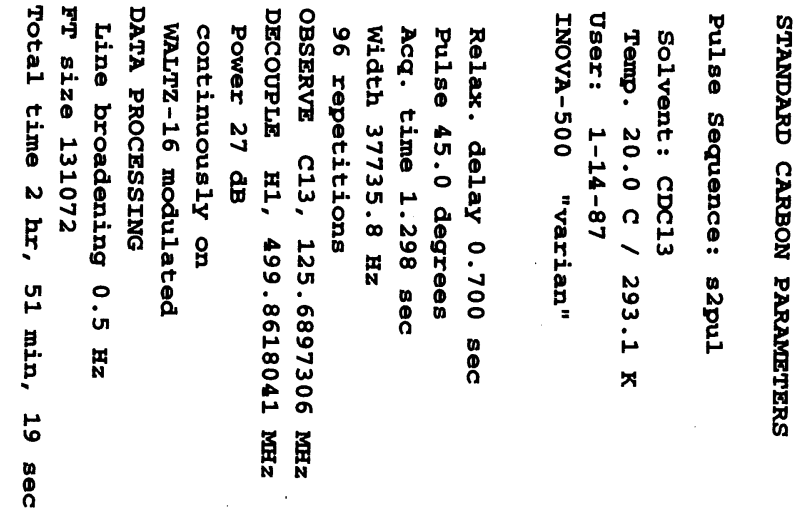

$\stackrel{\bullet}{\circ}$

홍

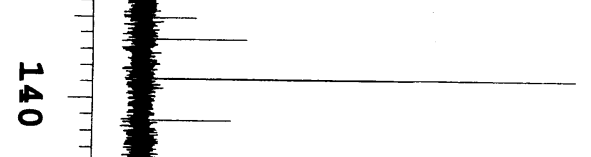

出当峷

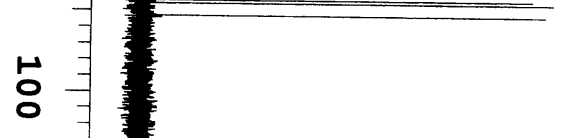

$\stackrel{\infty}{\circ}$

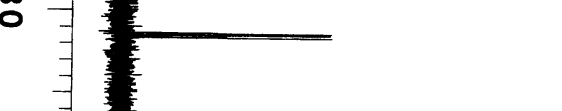

g

$\circ$ ㄱ․

点

N

$\circ$

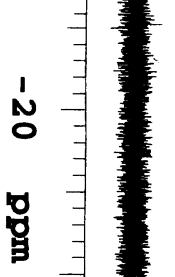

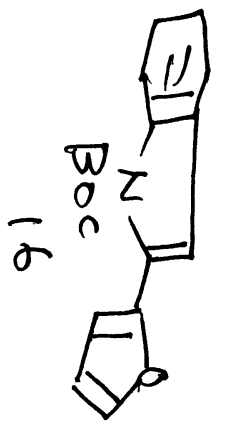




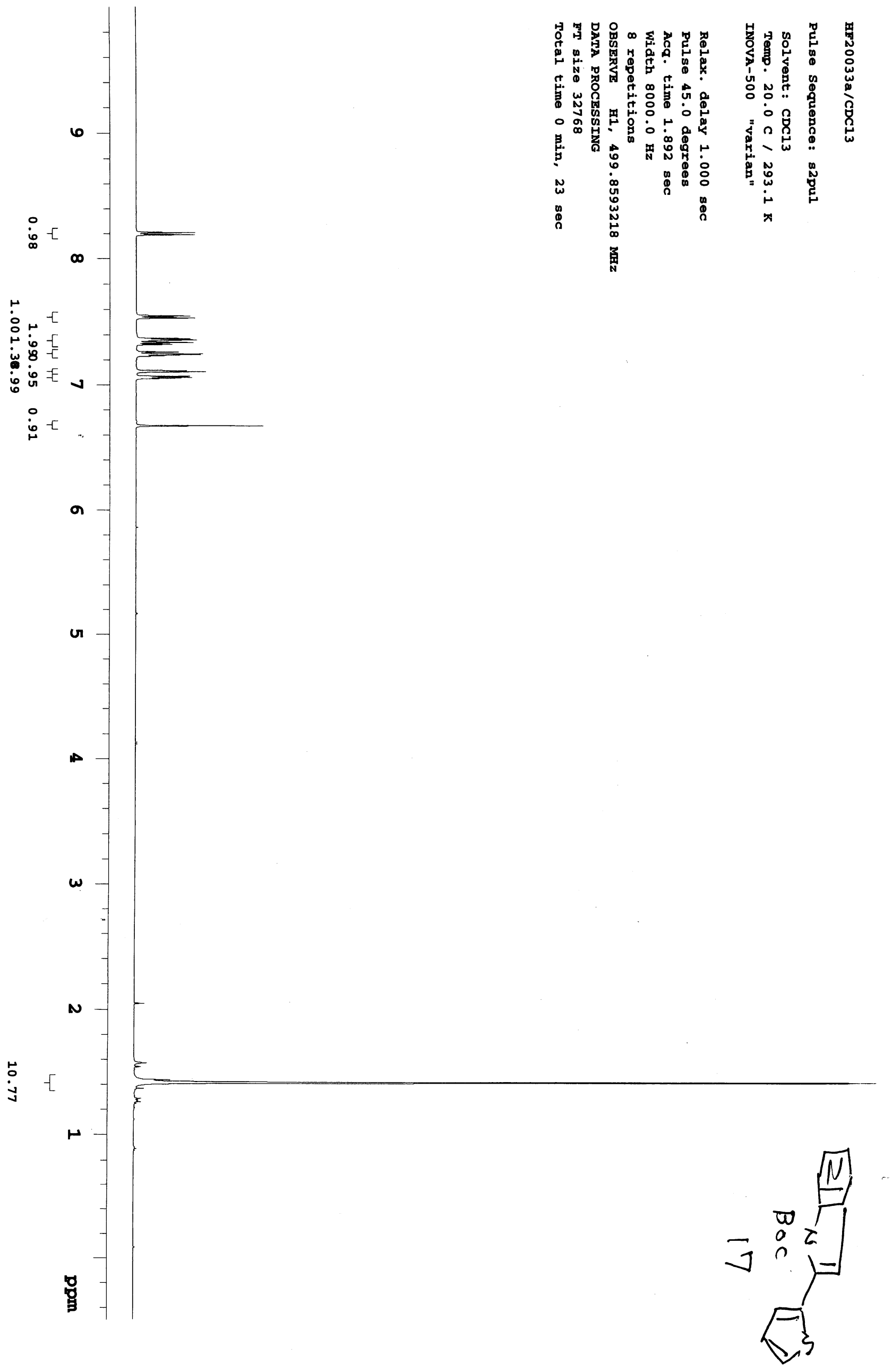




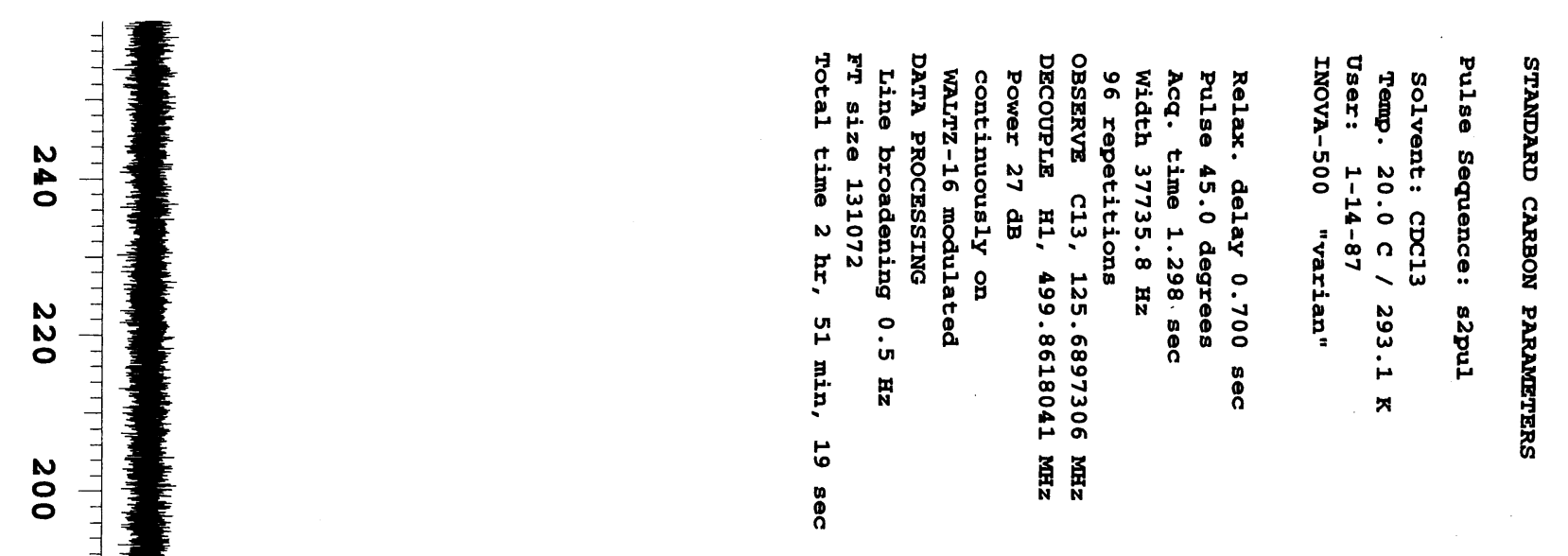

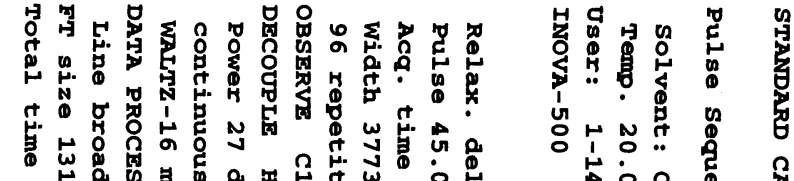

峁

응

占

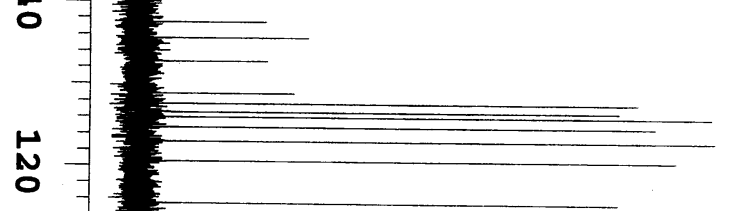

농

$\infty$

o

o

N

o

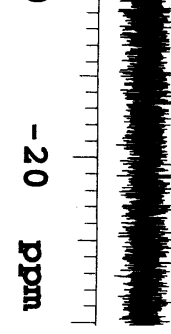

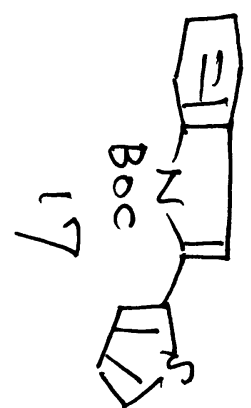




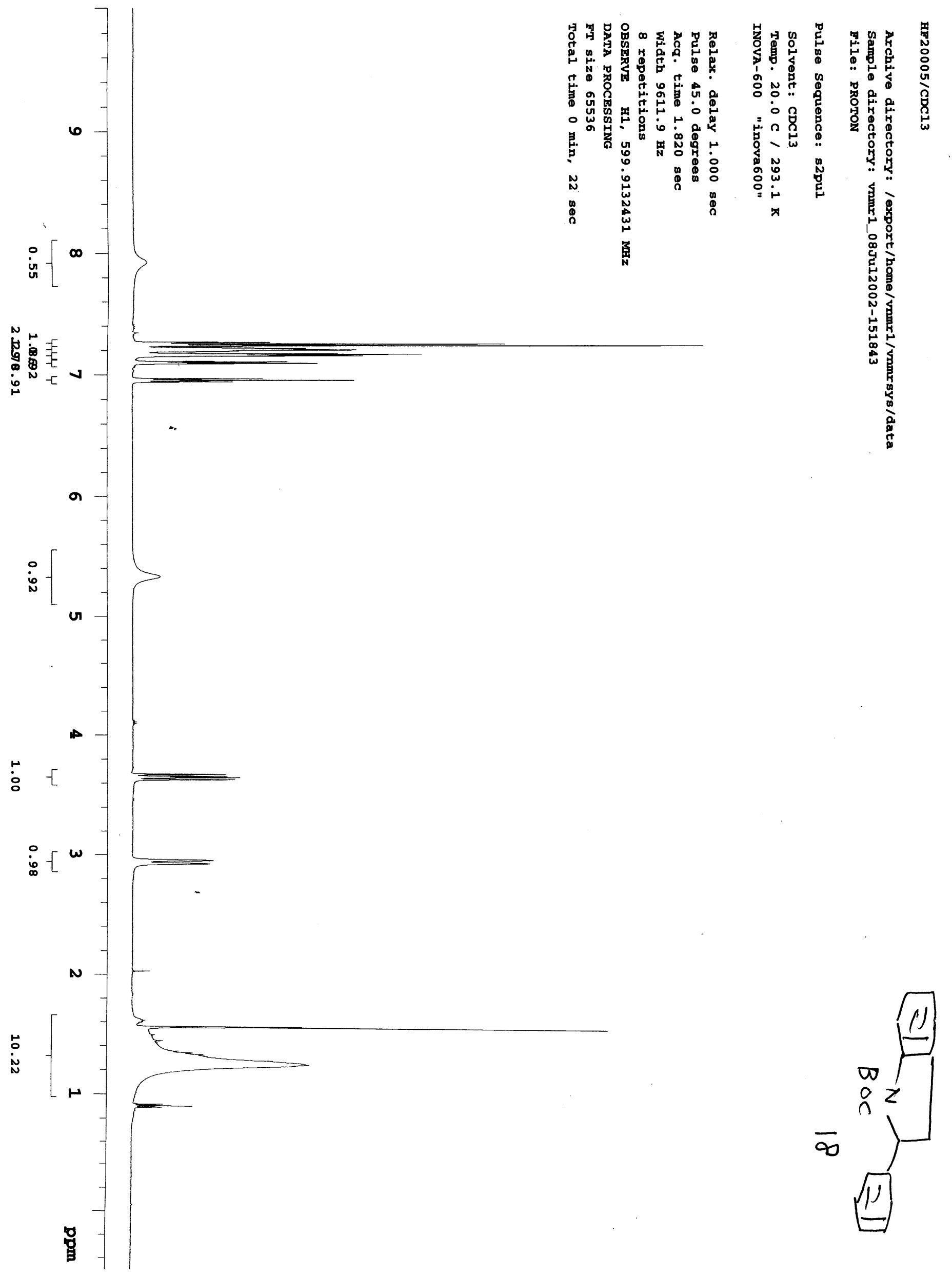




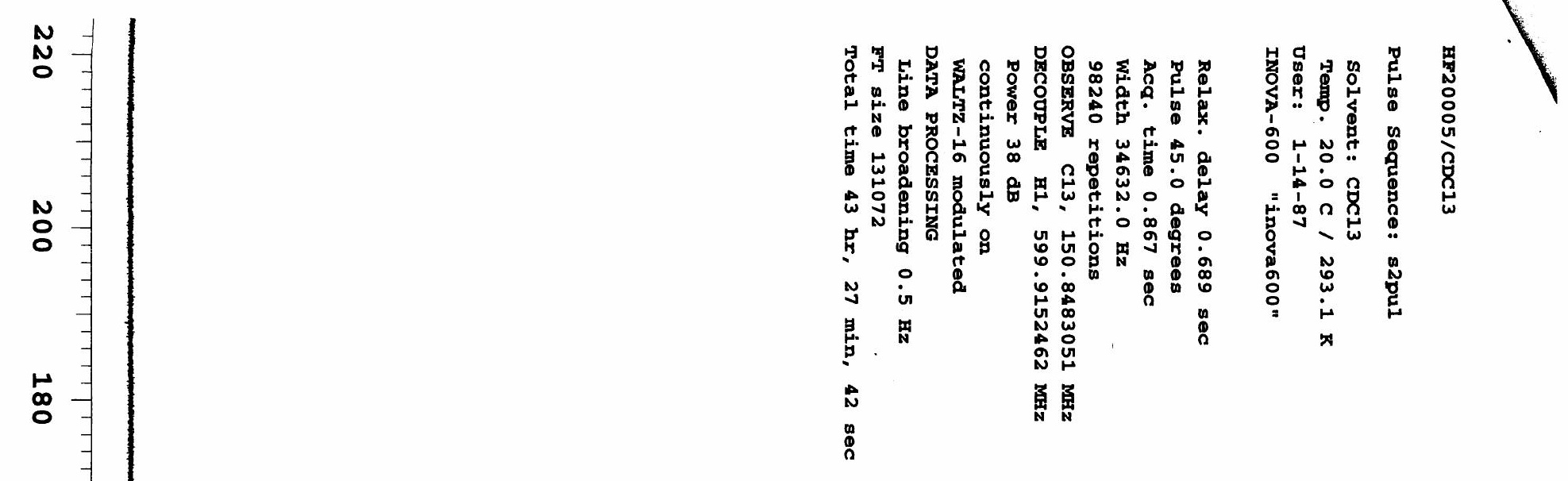

옹

占

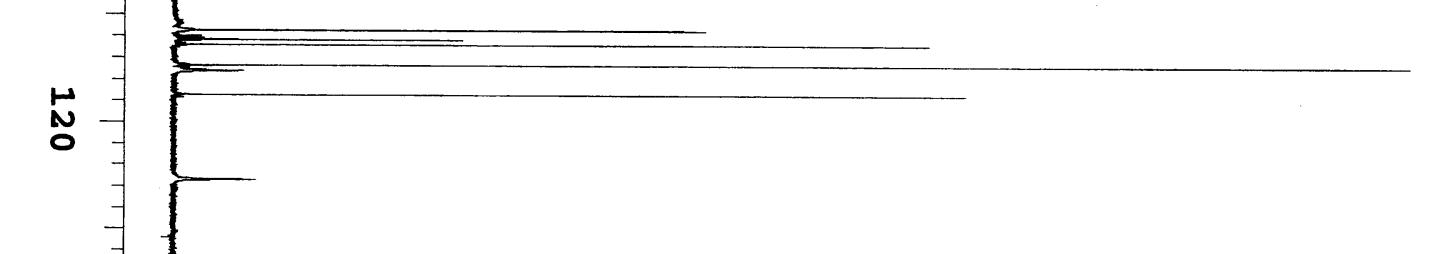

:

\&

0 -

의

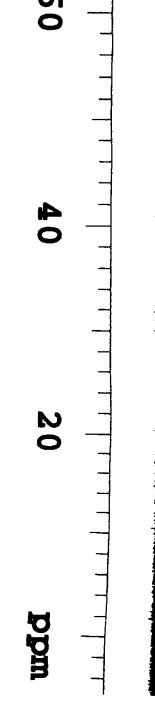

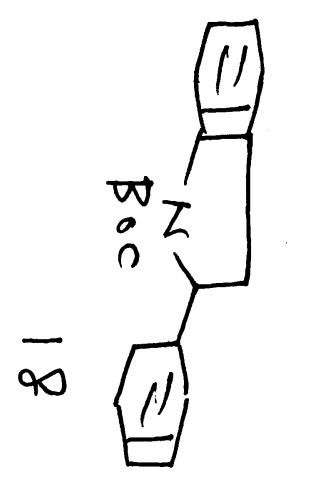




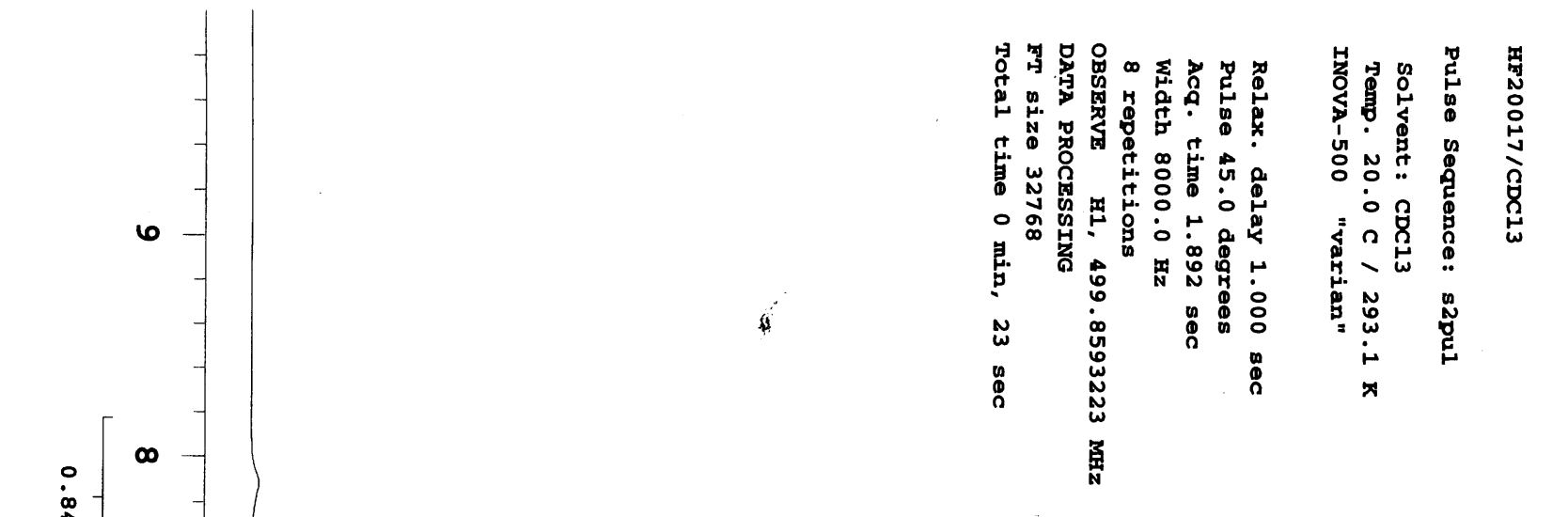

!n

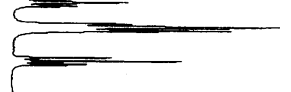

-

$\dot{0}^{N} \stackrel{\tilde{\omega}}{\omega}-[$

$\dot{:}-[$

$a$

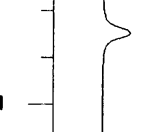

$r-$

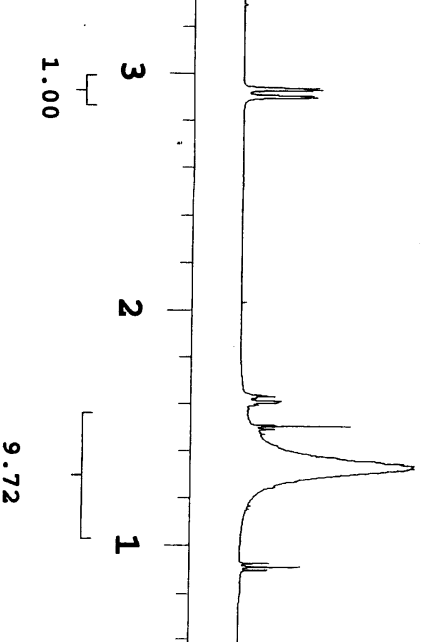

咱 

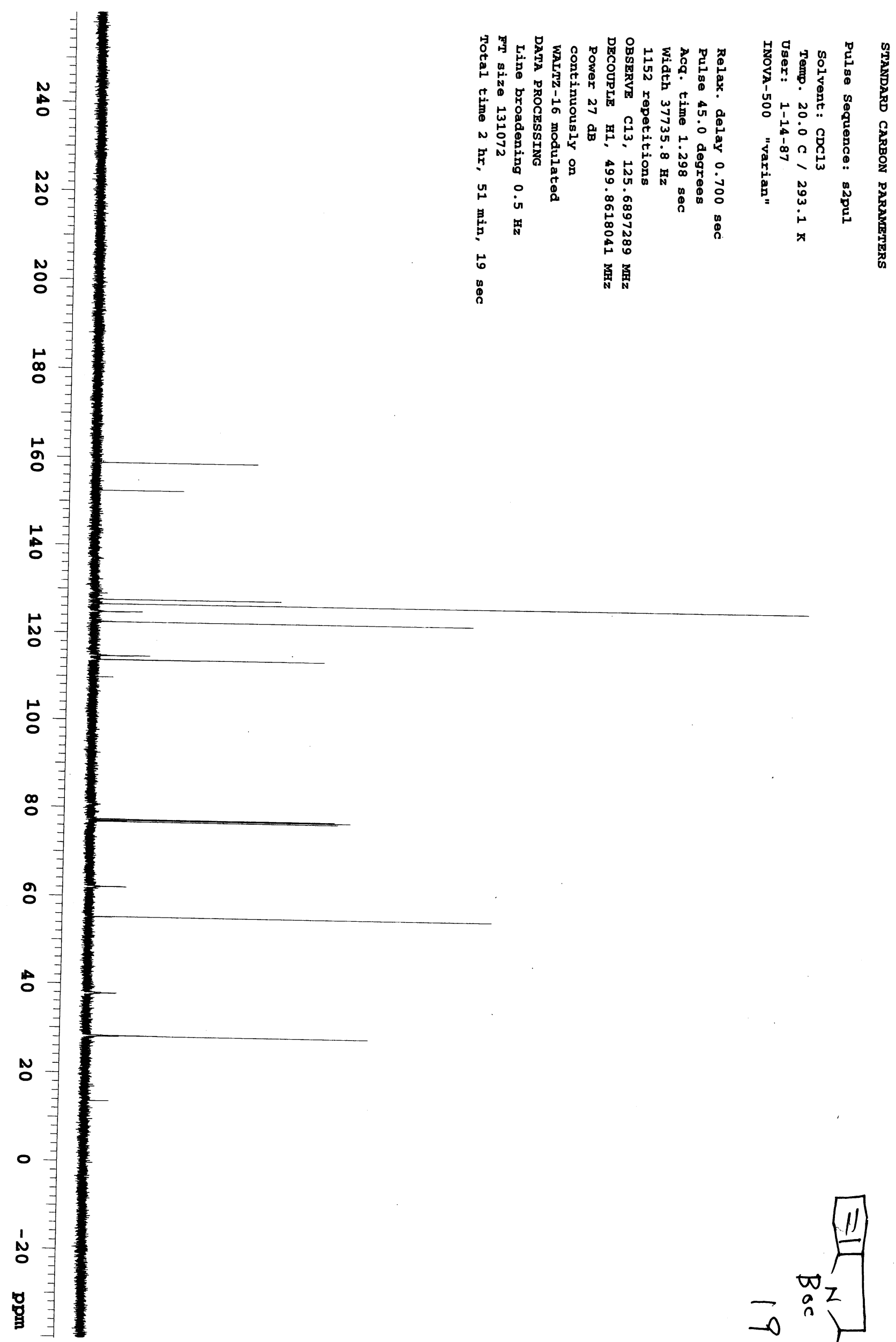

首兽

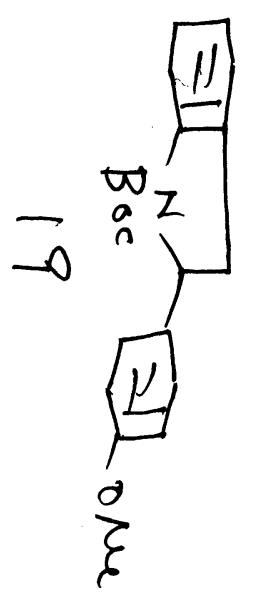



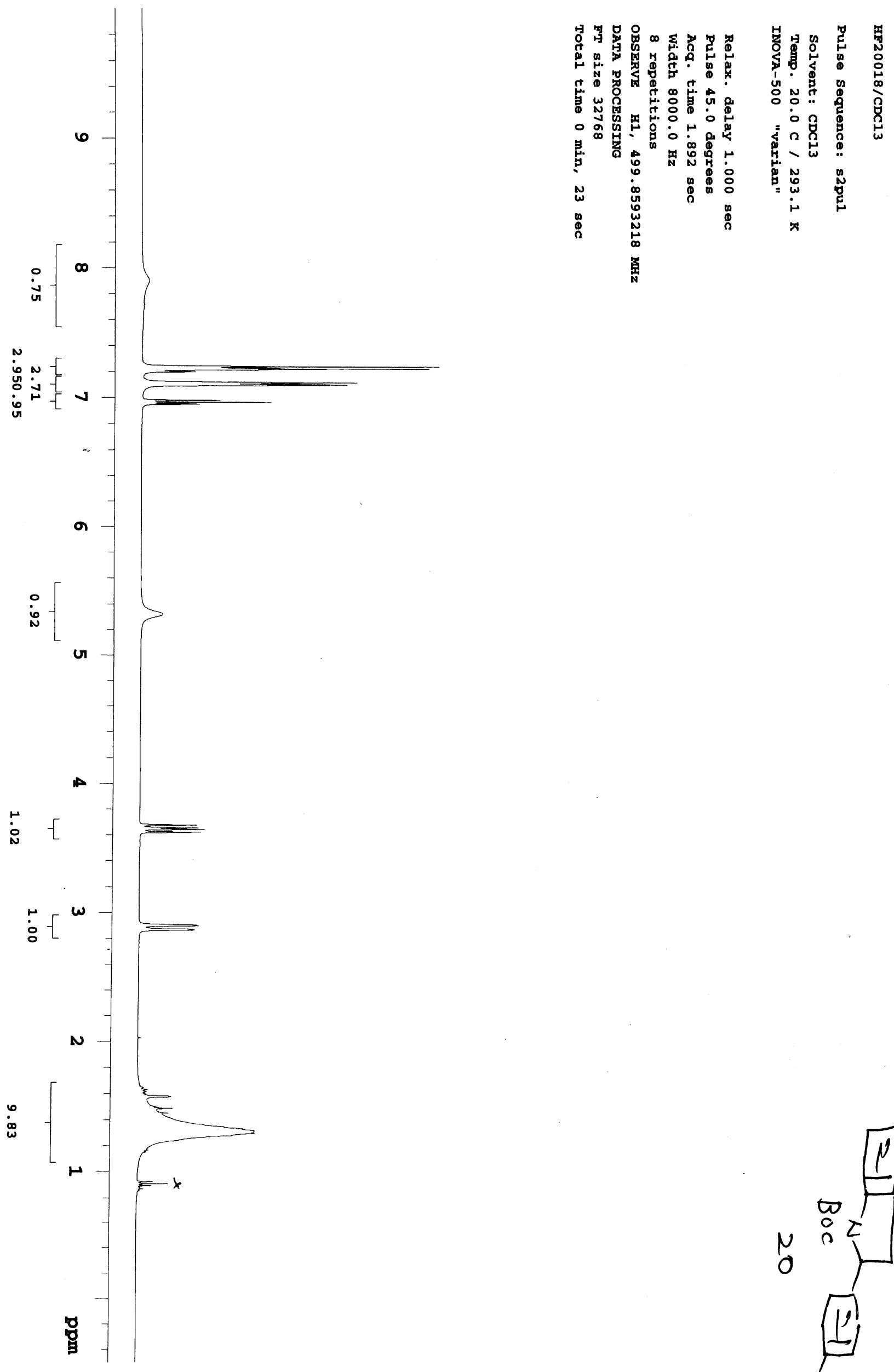

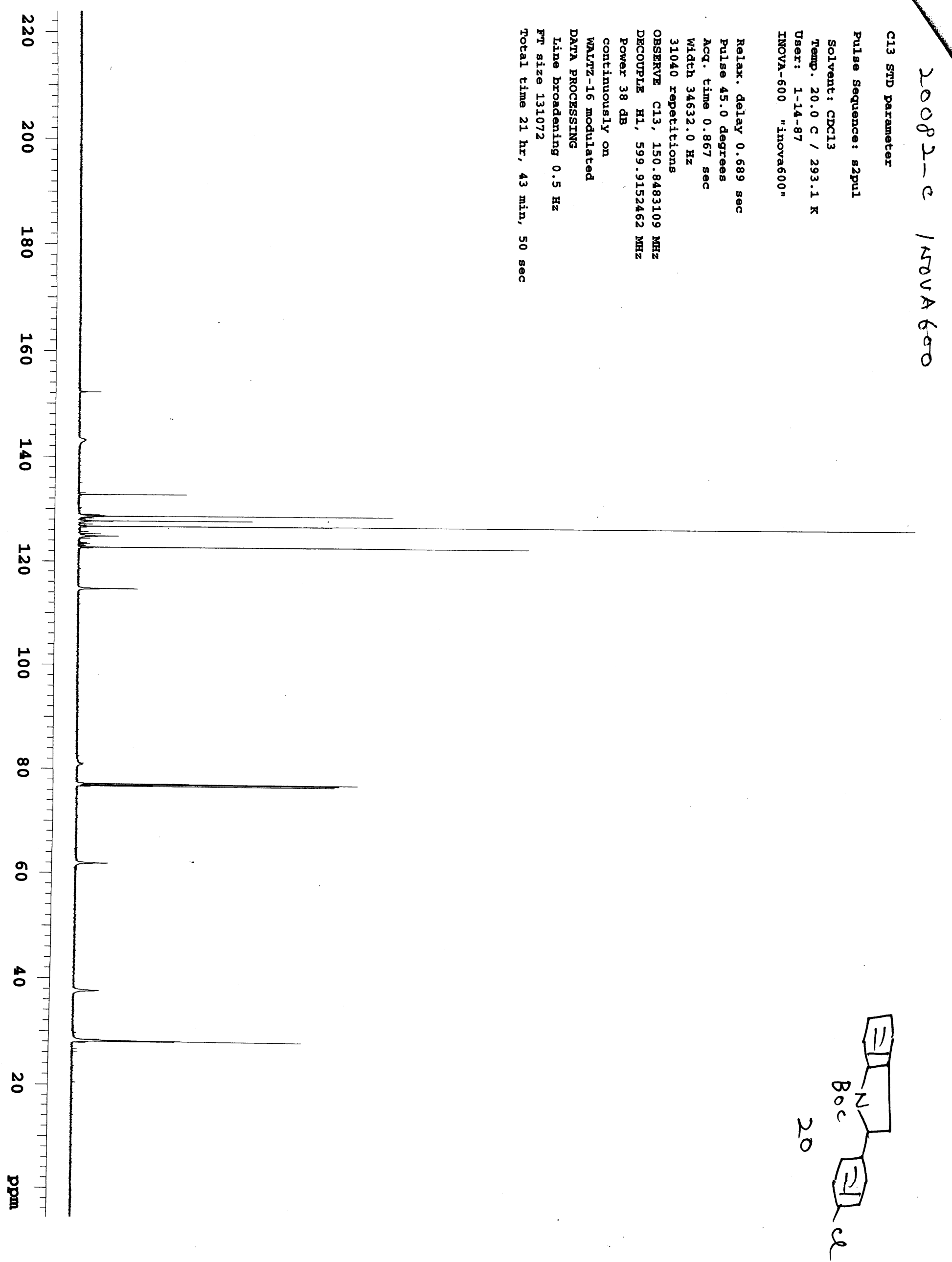


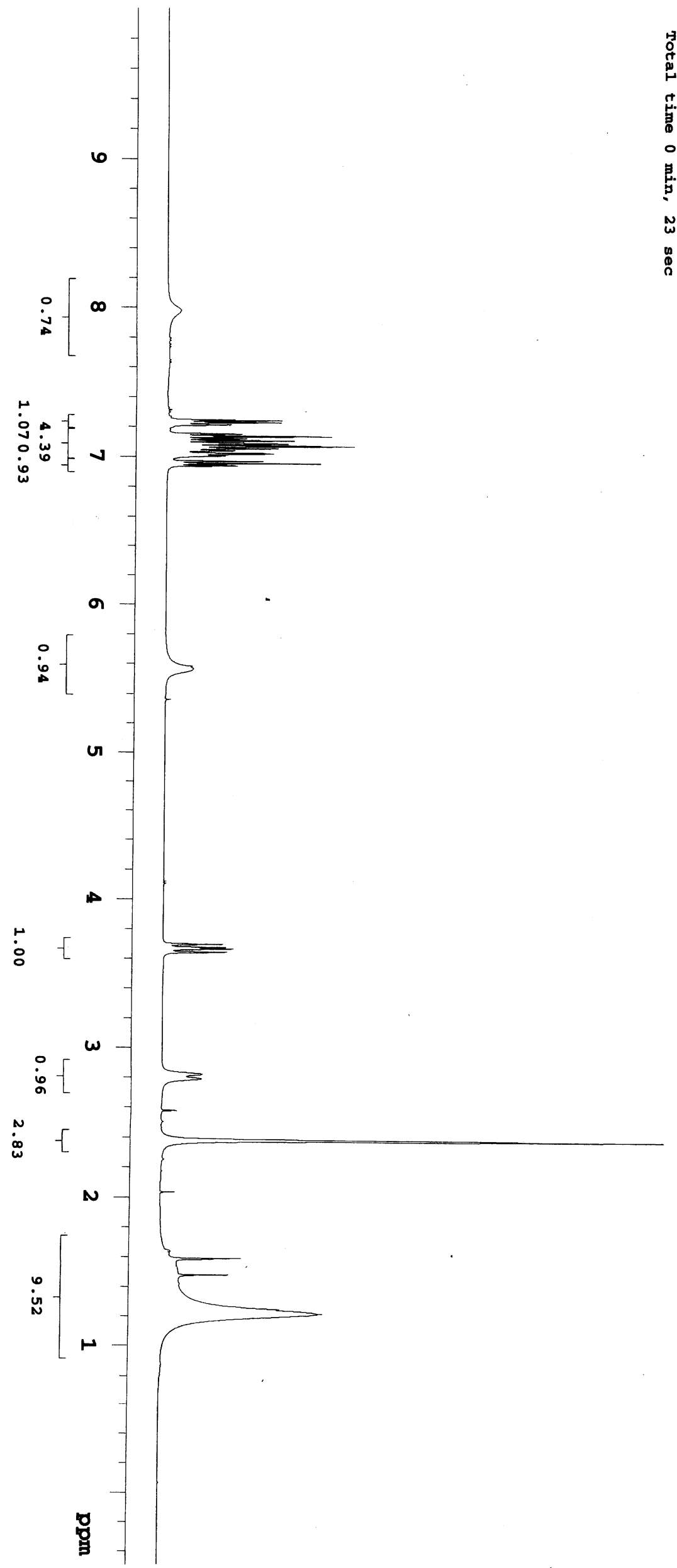

Mm

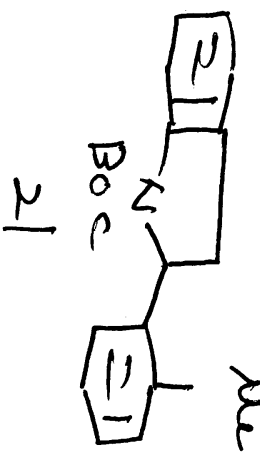




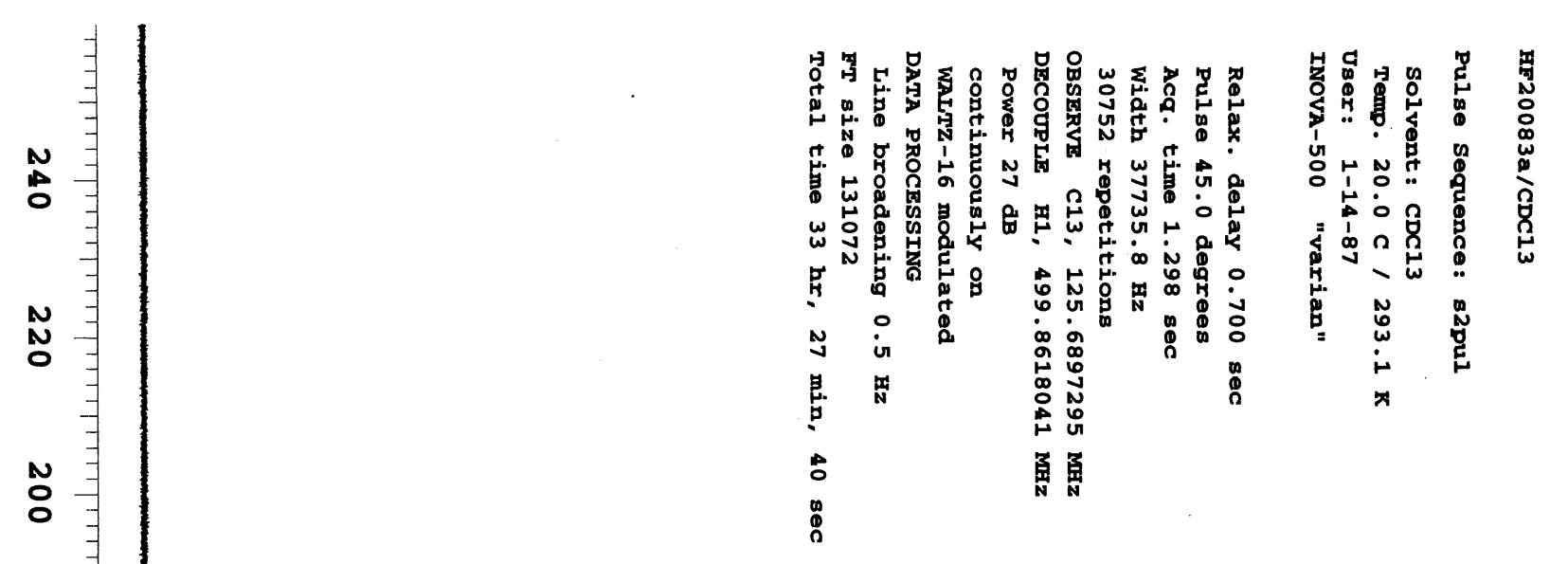

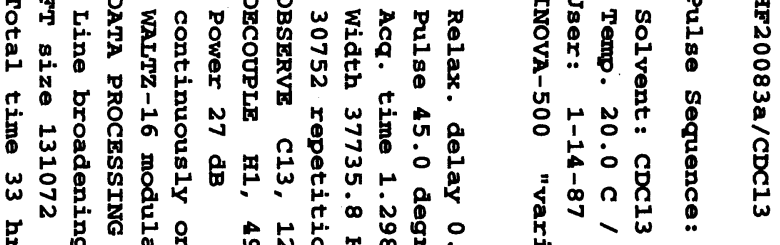

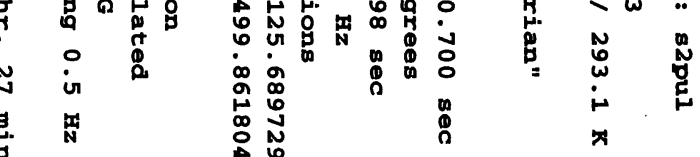

峞

뭉

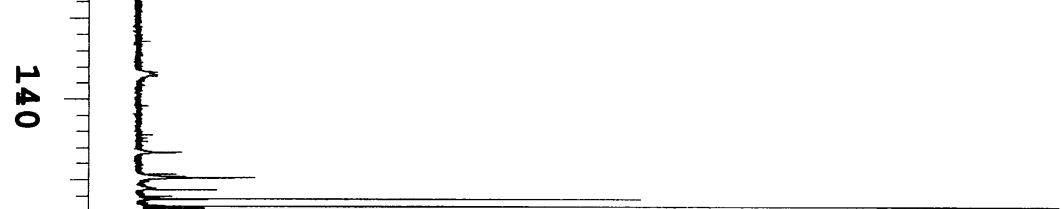

芯

:

ஃ

青

옹

\&

凊集

$\circ$

$:$

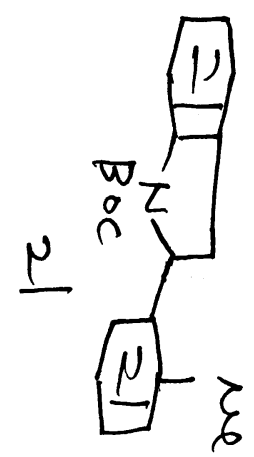




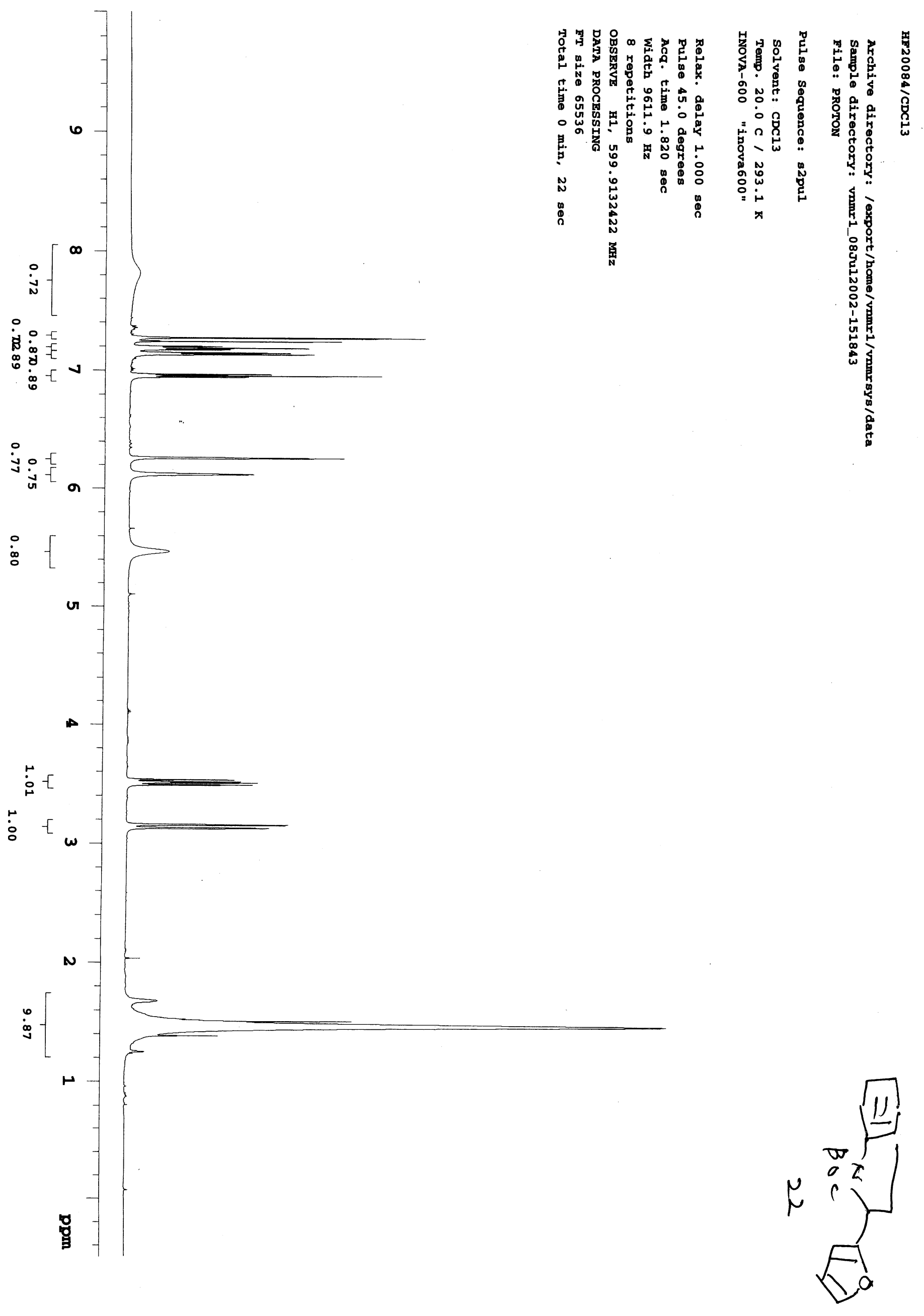



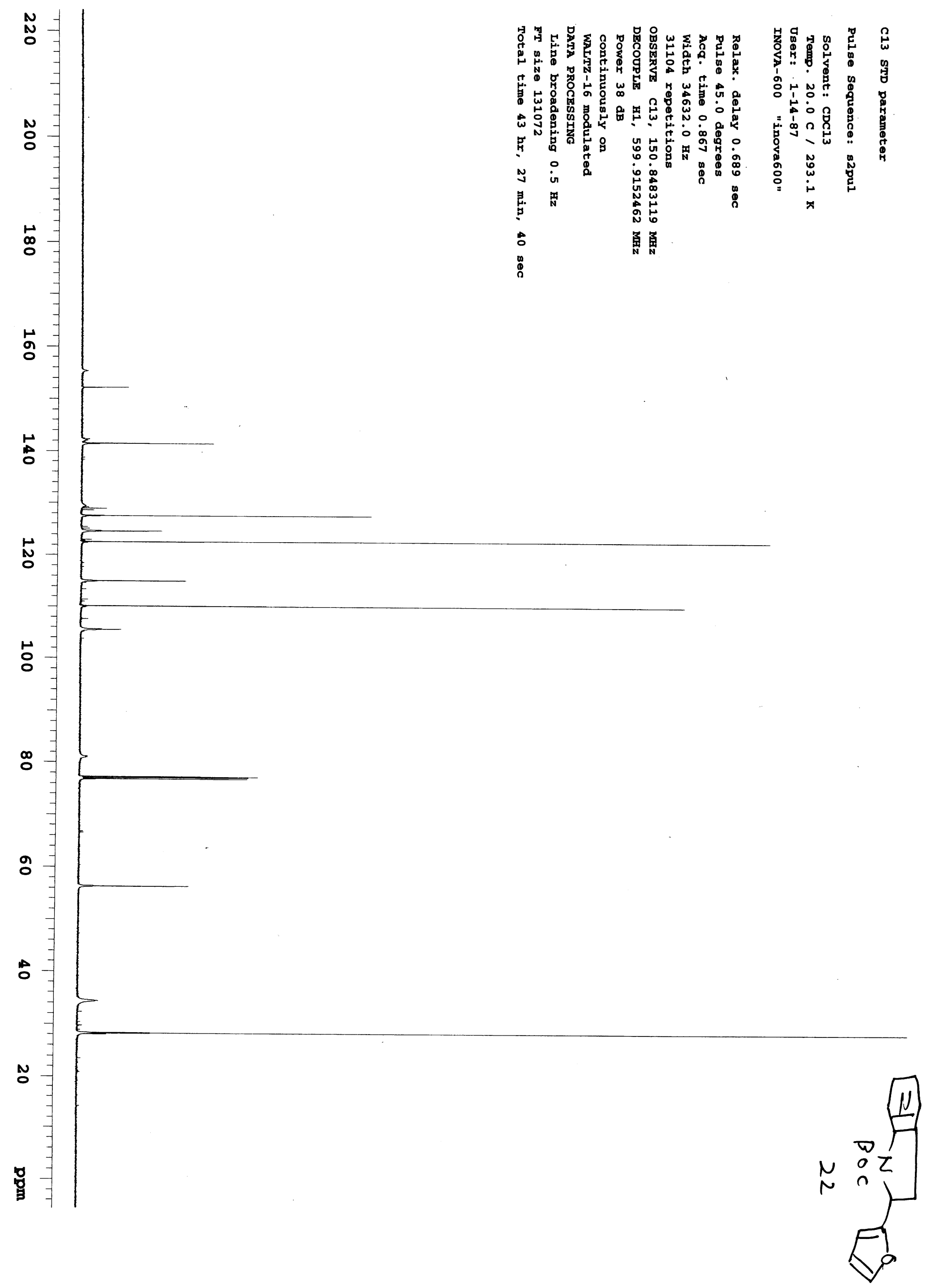